\title{
Novel Handover Optimization with a Coordinated Contiguous Carrier Aggregation Deployment Scenario in LTE-Advanced Systems
}

\author{
Ibraheem Shayea, ${ }^{1}$ Mahamod Ismail, ${ }^{1}$ Rosdiadee Nordin, ${ }^{1}$ Hafizal Mohamad, ${ }^{2}$ \\ Tharek Abd Rahman, ${ }^{3}$ and Nor Fadzilah Abdullah ${ }^{1}$ \\ ${ }^{1}$ Department of Electronics, Electrical and System Engineering, Faculty of Engineering and Build Environment, \\ Universiti Kebangsaan Malaysia, 43600 Bangi, Selangor, Malaysia \\ ${ }^{2}$ MIMOS Berhad, Technology Park Malaysia, 57000 Kuala Lumpur, Malaysia \\ ${ }^{3}$ Wireless Communication Center (WCC), Faculty of Electrical Engineering, Universiti Teknologi Malaysia (UTM), \\ 81310 Johor Bahru, Johor, Malaysia
}

Correspondence should be addressed to Ibraheem Shayea; shaia2009@yahoo.com

Received 28 September 2015; Revised 26 March 2016; Accepted 15 August 2016

Academic Editor: Lin Gao

Copyright ( 2016 Ibraheem Shayea et al. This is an open access article distributed under the Creative Commons Attribution License, which permits unrestricted use, distribution, and reproduction in any medium, provided the original work is properly cited.

\begin{abstract}
The carrier aggregation (CA) technique and Handover Parameters Optimization (HPO) function have been introduced in LTEAdvanced systems to enhance system performance in terms of throughput, coverage area, and connection stability and to reduce management complexity. Although LTE-Advanced has benefited from the CA technique, the low spectral efficiency and high pingpong effect with high outage probabilities in conventional Carrier Aggregation Deployment Scenarios (CADSs) have become major challenges for cell edge User Equipment (UE). Also, the existing HPO algorithms are not optimal for selecting the appropriate handover control parameters (HCPs). This paper proposes two solutions by deploying a Coordinated Contiguous-CADS (CCCADS) and a Novel Handover Parameters Optimization algorithm that is based on the Weight Performance Function (NHPOWPF). The CC-CADS uses two contiguous component carriers (CCs) that have two different beam directions. The NHPO-WPF automatically adjusts the HCPs based on the Weight Performance Function (WPF), which is evaluated as a function of the Signalto-Interference Noise Ratio (SINR), cell load, and UE's velocity. Simulation results show that the CC-CADS and the NHPO-WPF algorithm provide significant enhancements in system performance over that of conventional CADSs and HPO algorithms from the literature, respectively. The integration of both solutions achieves even better performance than scenarios in which each solution is considered independently.
\end{abstract}

\section{Introduction}

Several techniques and automatic functions have been proposed and developed to enhance system performance and reduce management complexity of Long Term Evolution Advanced (LTE-Advanced) systems, Releases (Rel.) 10 to 13. Carrier aggregation is a technique that was proposed to enhance system throughput and provide a wider coverage area [1-4], while the Self-Optimization (SO) is one of the SelfOrganization Network (SON) features that were introduced in LTE [5] and LTE-Advanced [6-11] systems. The main aim of Self-Optimization is to automate the management process by dynamically adapting system parameters to improve system quality. It also manages the network complexity that is a result of the significant increases in the size and complexity of modern mobile cellular systems.

Five CADSs have been introduced with the advent of CA technique [1-4] in LTE-Advanced systems by the Third Generation Partnership Project (3GPP). These CADSs have been introduced to support UE's mobility and enhance system performance through the UE mobility in the cells. Each CADS provides a different coverage area, which depends 
on the operating frequency and the beam directions of the configured CCs. Therefore, each CADS provides different system performance results for mobile UEs. Thus, if a CA technique is considered, one of these scenarios should be carefully selected via a mobility study. Because CADS-4 and CADS- 5 represent repeated scenarios of CADS-1 and CADS3 , this paper will focus on only the first three CADSs. In CADS-1, both CCs provide the same coverage, which is supporting the UE's mobility, but overlaying the CCs leads to insufficient coverage at the boundaries of both cells. In CADS-2, only CC1 provides sufficient coverage, whereas CC2 provides a smaller coverage and is overlaid on $\mathrm{CC} 1$. Therefore, the coverage at the cell boundaries of $\mathrm{CCl}$ will be insufficient. In CADS-3, only $\mathrm{CC} 1$ provides sufficient coverage, which leads to insufficient coverage at the cell boundaries of each CC even if CC2 is directed at the cell boundary of CC1. Although several CADSs have been introduced in LTEAdvanced systems [1-4], issues related to low throughput and high outage probability have yet to be solved. These issues may due to insufficient coverage provided by the serving Evolved Node B (eNB). Thus, a new CA deployment scenario is needed to provide sufficient and equal coverage for the served eNB.

In the field of SONs, the HPO is an important SO function that was introduced in LTE systems from Rel. 9 to Rel. 13 [6-11] to dynamically adapt HCPs to handle handover problems. Handover is required to support UE mobility in the coverage area and is performed by switching the radio connection links of the UE from the serving cells to the target cells. Thus, suboptimal settings of HCPs may lead to large numbers of unnecessary handovers, such as high handover ping-pong probability (HPPP), high Handover Failure Probability (HFP), and high Radio Link Failure (RLF). These lead to wasted network resources. Therefore, the main objective of introducing HPO function is to reduce the number of HPPP, HFP, and RLF events that may result from the suboptimal tuning of HCPs. In addition, HPO function attempts to decrease the wasteful usage of system resources due to needless optimization for HCPs. Although the road map of the conventional HPO was introduced and developed to reduce handover problems, it is not the optimal algorithm for optimizing HCPs. Therefore, several handover algorithms have been developed to optimize HCPs [12-14]. The Weighted Performance based on Handover Parameter Optimization (WPHPO) algorithm adaptively tunes HCPs based on the average Handover Performance Indicator (HPI), which is evaluated as a function of the HFP, HPPP, and Drop Call Probability (DCP) [12, 13]. The Fuzzy Logic Controller (FLC) was proposed to adaptively modify the handover margin (HOM) level while setting the Time-ToTrigger (TTT) to a fixed value [14]. The FLC adjusts the HOM level based on two control input parameters, which are known as DCP and Handover Ratio (HOR). Although the conventional HPO, WPHPO, and FLC algorithms contribute to enhancing the handover performance for UEs, nonrobust and nonoptimal algorithms for selecting appropriate HCPs over CC-CADS exist. Consequently, an optimal HPO algorithm is needed for the CA technique in LTE-Advanced systems.
This paper proposes two enhancement solutions by deploying appropriate CC-CADS and NHPO-WPF algorithm. The CC-CADS uses two CCs that operate on two contiguous frequency bands, with one transmitting antenna of each CC. The beam of $\mathrm{CC} 1$ is directed at the cell boundary of CC2 and the beam of CC2 is directed at the cell boundary of CC1. The NHPO-WPF algorithm estimates the suitable HCP values based on a WPF, which estimates the optimization level based on three bounded functions. These three functions are evaluated as a function of (i) the SINR, (ii) the cell load, and (iii) the UE's velocity. The NHPO-WPF algorithm can adaptively adjust the HCPs values for each UE independently based on these three parameters. Therefore, suitable HCPs values will be selected, which leads to taking an intact handover decision to the suitable target eNB at the fit time, which in turn leads to decreased HPPP, HFP, and RLF. Thus, the CC-CADS and NHPO-WPF algorithm will contribute to effectively supporting seamless connectivity between the UE and the serving network.

The remainder of this paper is organized as follows. Section 2 describes the background and related work, and Section 3 presents the proposed solutions. The system model is described in Section 4, the evaluation of the handover performance is presented in Section 5, and the results are discussed in Section 6. Section 7 concludes the paper.

\section{Background and Related Work}

2.1. Standard Carrier Aggregation Deployment Scenarios. Figure 1 shows the first three CADSs (i.e., CADS-1, CADS-2, and CADS-3), which were introduced in [1-4]. In CADS-1, the operating frequencies for $\mathrm{CC} 1$ and $\mathrm{CC} 2$ are assumed to lie in a contiguous band, while the beams of both CCs are assumed to be directed in the same direction. Therefore, the coverage of CC1 and CC2 overlap and are colocated, as shown in Figure 1(a), and provide nearly the same coverage area. In CADS-2, the frequencies of CC1 and CC2 are assumed to operate on different bands; $\mathrm{CC} 1$ is assumed to operate in the lower frequency band, and CC2 is assumed to operate in the higher frequency band. In addition, the beams of both CCs are assumed to be directed in the same direction. Therefore, the coverage of the CC1 and CC2 cells is overlaid and colocated, as shown in Figure 1(b), but CC1 has a larger coverage area than CC2 due to the smaller path loss that results from $\mathrm{CC} 1$. Therefore, only $\mathrm{CC1}$ provides sufficient coverage, and CC2 is used to extend the bandwidth to provide higher throughput to the UEs. In CADS-3, CC1 and CC2 are assumed to operate on noncontiguous bands; $\mathrm{CC} 1$ is assumed to operate on the lower frequency band, and CC2 is assumed to operate in the higher frequency band. The beams of the CCs are assumed to be directed in different directions. Therefore, the coverage areas of $\mathrm{CC} 1$ and $\mathrm{CC} 2$ are colocated as shown in Figure 1(c), but $\mathrm{CC1}$ has a larger coverage area than CC2 due to the smaller path loss that results from CC1.

In the first CADS, both CCs can provide sufficient coverage but are overlaid. Therefore, the coverage provided by both CCs is focused in one direction and is insufficient everywhere around the serving cell, especially at the cell 


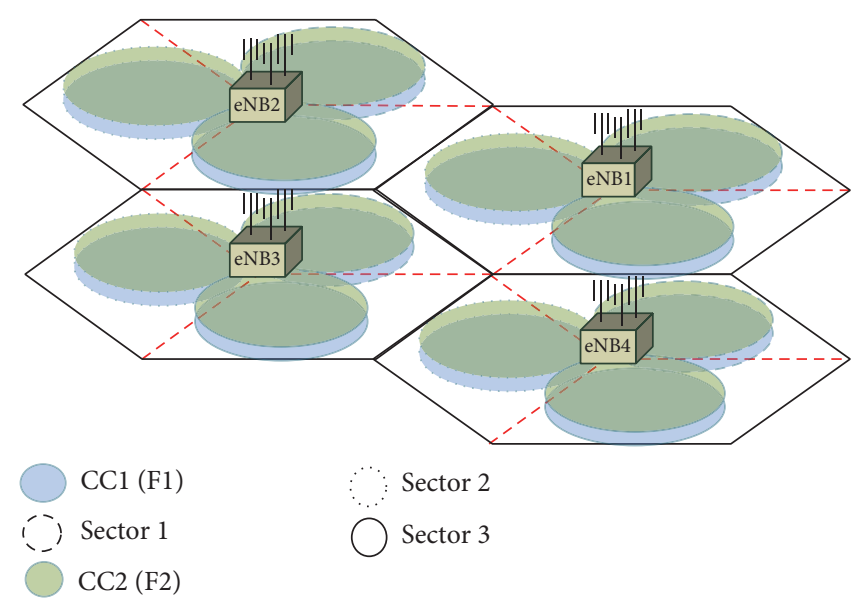

(a) CADS-1

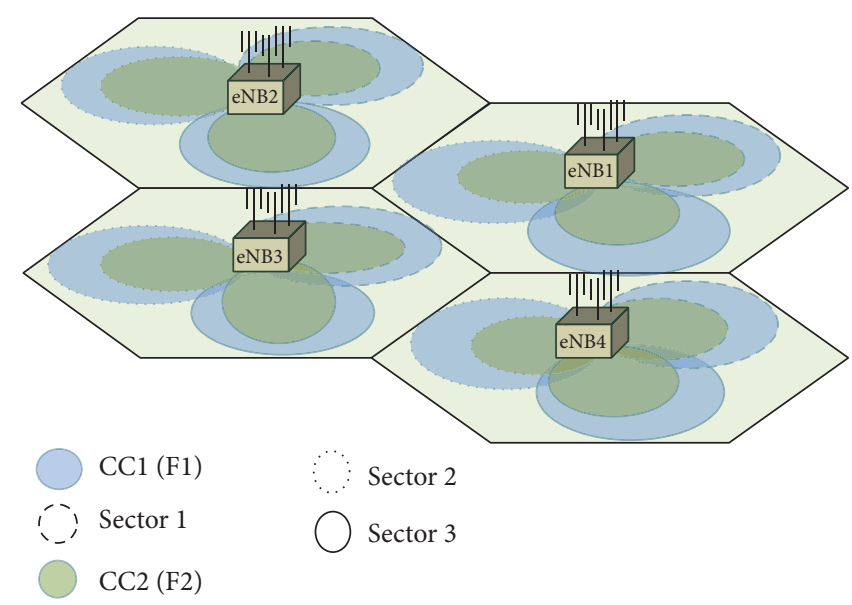

(b) CADS-2

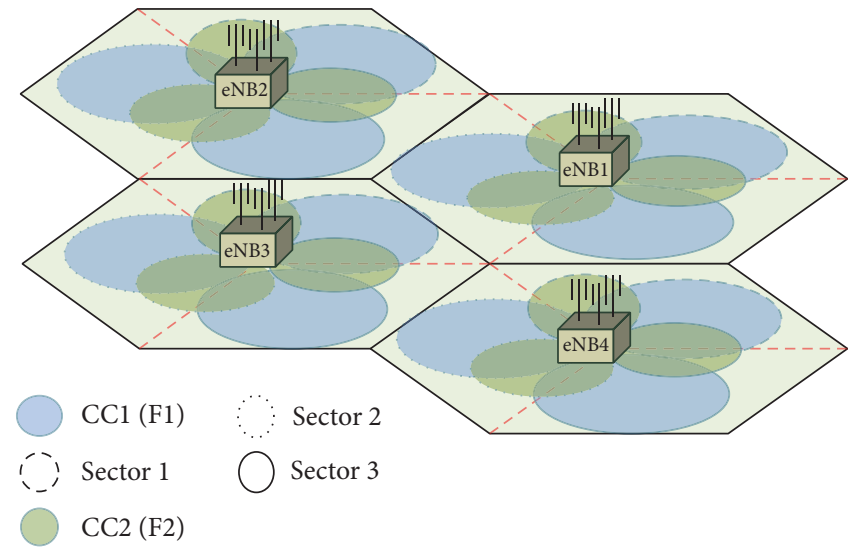

(c) CADS-3

Figure 1: Three different CADSs that have been standardized by the 3GPP [1-4].

boundaries of the CCs. In CADS-2, only $\mathrm{CC} 1$ can provide sufficient coverage, whereas CC2 provides a smaller coverage and is overlaid on CCl. The coverage is insufficient at the cell boundaries of CC1. In CADS-3, only $\mathrm{CCl}$ can provide sufficient coverage; $\mathrm{CC} 2$ provides insufficient coverage due to the large path loss produced by CC2. Therefore, the coverage provided by both CCs will be insufficient at the cell boundaries of each CC. These three CA deployment scenarios cannot provide sufficient coverage everywhere around the serving eNB. A new CA deployment scenario is thus needed to provide sufficient and equal coverage around the serving eNB.

2.2. Handover Parameter Optimization Studies. The road map of HPO function (conventional HPO algorithm) was introduced by the $3 \mathrm{GPP}$ as a fundamental feature to deploy LTE-Advanced systems [5-11, 16]. HPO aims to adaptively adjust the HCPs values to maintain system quality and perform automatic optimization for HCPs with minimal human intervention. In particular, the HPO function attempts to detect and perform corrections of (i) RLF due to mobility and (ii) the ping-pong effect. The conventional
HPO algorithm adaptively adjusts the HCPs when RLF or ping-pong is detected as a result of (i) an early handover, (ii) a late handover, (iii) a handover to the wrong cell, or (iv) inefficient use of system resources caused by an unnecessary handover. These outcomes occur as a result of suboptimal HCP settings. Thus, if RLF or HPPP is detected as a result of suboptimal HCPs settings, the HPO algorithm can adjust the HCPs values for the corresponding cell to solve the handover problem. Although the conventional HPO was developed to reduce handover problems, it is not the optimal algorithm for optimizing HCPs. Therefore, several handover parameter optimization studies have been conducted to address the drawbacks of the conventional HPO algorithm in LTE systems, and several solutions have been proposed to handle handover problems that are caused by a suboptimal optimization (see [12-14] and references therein). These solutions will be highlighted and investigated in this paper to compare their performance with that of the proposed algorithm. The conventional HPO algorithm will also be considered to show the superiority of the proposed algorithm.

WPHPO was proposed to adaptively tune HCPs for cells based on the average HPI $[12,13]$. HPI is evaluated 


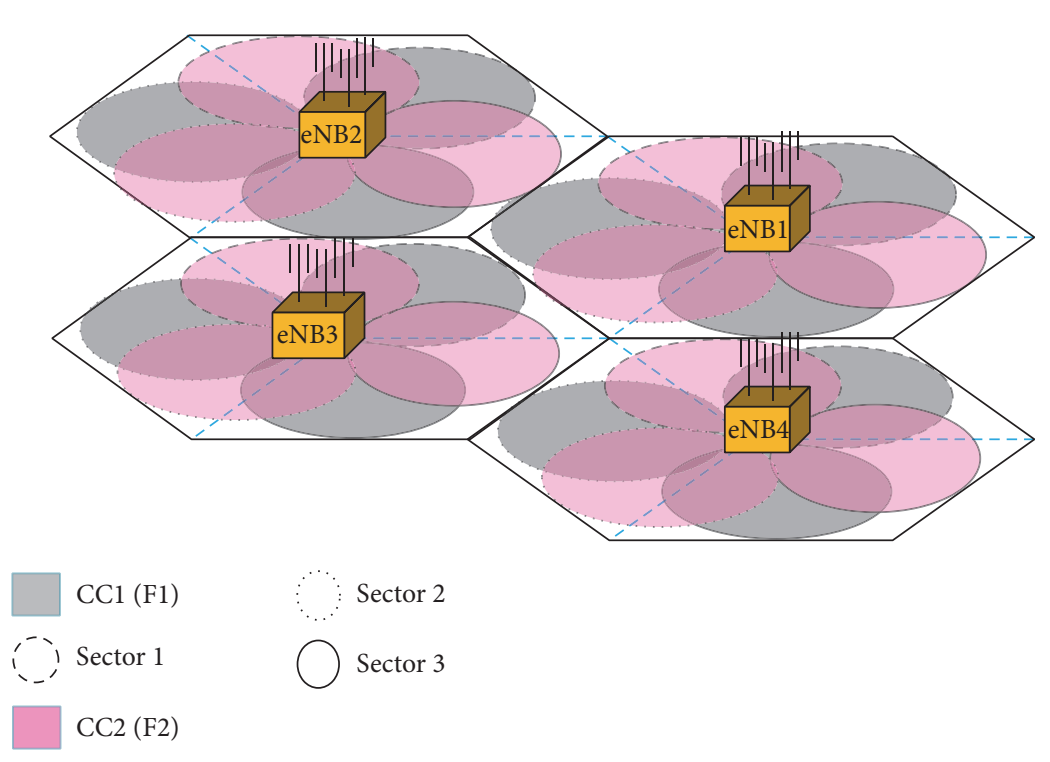

Figure 2: Coordinated Contiguous-Carrier Aggregation Deployment Scenario.

as a function of HFP, HPPP, and DCP. However, WPHPO attempts to find the suitable HOM level and TTT interval for each cell. When the HPI at time $\tau+\rho$ becomes greater than the HPI at time $\tau$, the system performance is degraded, while if the HPI at time $\tau+\rho$ becomes smaller than HPI at time $\tau$, it indicates that the cell performance is good. Therefore, if the differences between $\operatorname{HPI}(\tau)$ and $\operatorname{HPI}(\tau+\rho)$ become equal to or greater than a specific level, the WPHPO performs a one-step optimization. Otherwise, the WPHPO will continue using the older handover parameter values.

FLC was proposed to adaptively modify the HOM level, while the TTT interval is assumed to be fixed at $100 \mathrm{~ms}$ [14]. However, the FLC adjusts the HOM level based on two control input parameters, which are known as Call Drop Rate (CDR) and HOR. Based on these two input parameters, the FLC automatically performs the optimization to select the suitable HOM level. The HOM level is selected for each cell based on the CDR and HOR levels in the corresponding cell. FLC adjusts the HOM in every Transmission Time Interval (TTI), and the selected HOM level is restricted between 0 and $12 \mathrm{~dB}$.

These HPO algorithms were aimed at providing efficient optimization for HCPs, but no optimal solution exists. All the highlighted HPO algorithms perform optimization for all UEs in the cell simultaneously. This leads to an increased probability of unnecessary handovers by adjusting the HCP values for UEs who do not need their HCPs to be optimized. In addition, some of these algorithms, such as FLC, adjust only the HOM level, while the TTT is set to a fixed value. This malfunction reduces the main purpose of the HPO function. Consequently, nonrobust and nonoptimal algorithms for selecting appropriate HCPs over CC-CADS have been developed. Moreover, handover parameter optimization with the existing CA technique is one of the most significant issues that should be investigated and validated in current research on LTE-Advanced systems. Developing the HPO algorithm that was used in Rel. 8, 9, and 10 was necessary for Rel. 11. Therefore, a new solution to overcome the shortcomings of the conventional and the existing HPO algorithm from the literature is needed.

\section{Proposed Solutions}

In this paper, novel CC-CADS and NHPO-WPF algorithm are proposed to enhance the system performance with the existing CA technique in the LTE-Advanced system. These two solutions are briefly described in the following two subsections.

3.1. Coordinated Contiguous-Carrier Aggregation Deployment Scenario (CC-CADS). In this paper, a new carrier aggregation deployment scenario is proposed and introduced as Coordinated Contiguous-Carrier Aggregation Deployment Scenario (CC-CADS). This proposed deployment scenario, CC-CADS, considers two CCs to be configured in the system. Both CCs are assumed to be colocated and operated on two frequencies in a contiguous band. Meanwhile, the beam of each configured CC is proposed to be pointed in a different direction; the beam of $\mathrm{CC} 1$ is directed to the sector center, and the beam of CC2 is directed toward the cell boundary of $\mathrm{CC} 1$ as shown in Figure 2. In addition, more details about CC-CADS as compared to the existing CADS are illustrated in Table 1. Therefore, the CC-CADS will combine the features of CADS- 1 and CADS- 3 as long as the CC1 and CC2 are in a contiguous band and their beams are directed in different directions. Thus, the proposed CC-CADS is expected to offer sufficient coverage than the previous CADS deployment discussed earlier in Section 2. Meanwhile, it is expected that both CCs can be aggregated at the same eNB. Because CC1 and CC2 operate in a contiguous band, the coverage areas that are supported by the two CCs will be sufficient and nearly 
TABLE 1: Summary of the proposed CC-CADS and the previous CADS from the literature [1-4].

\begin{tabular}{lcccc}
\hline & CADS-1 & CADS-2 & CADS-3 & Proposed CC-CADS \\
\hline Band type & Contiguous & Non-Contiguous & Non-Contiguous & Contiguous \\
Frequency of CC1 & $2 \mathrm{GHz}$ & $2 \mathrm{GHz}$ & $2 \mathrm{GHz}$ & $2 \mathrm{GHz}$ \\
Frequency of CC2 & $2.0203 \mathrm{GHz}$ & $3.5 \mathrm{GHz}$ & $3.5 \mathrm{GHz}$ & $2.0203 \mathrm{GHz}$ \\
Spacing frequency & $300 \mathrm{kHz}$ & $300 \mathrm{kHz}$ & $300 \mathrm{kHz}$ & $300 \mathrm{kHz}$ \\
Beam orientation & Both CCs have the & Both CCs have the & Each CC has different & Each CC has different \\
& same direction & same direction & direction & direction \\
Coverage areas & Identical & Overlaid and & Co-located & Co-located \\
Initial PCC & CC1 & CCl & CC1 & CC2 \\
Initial SCC & CC2 & CC2 & & CC2 \\
\hline
\end{tabular}

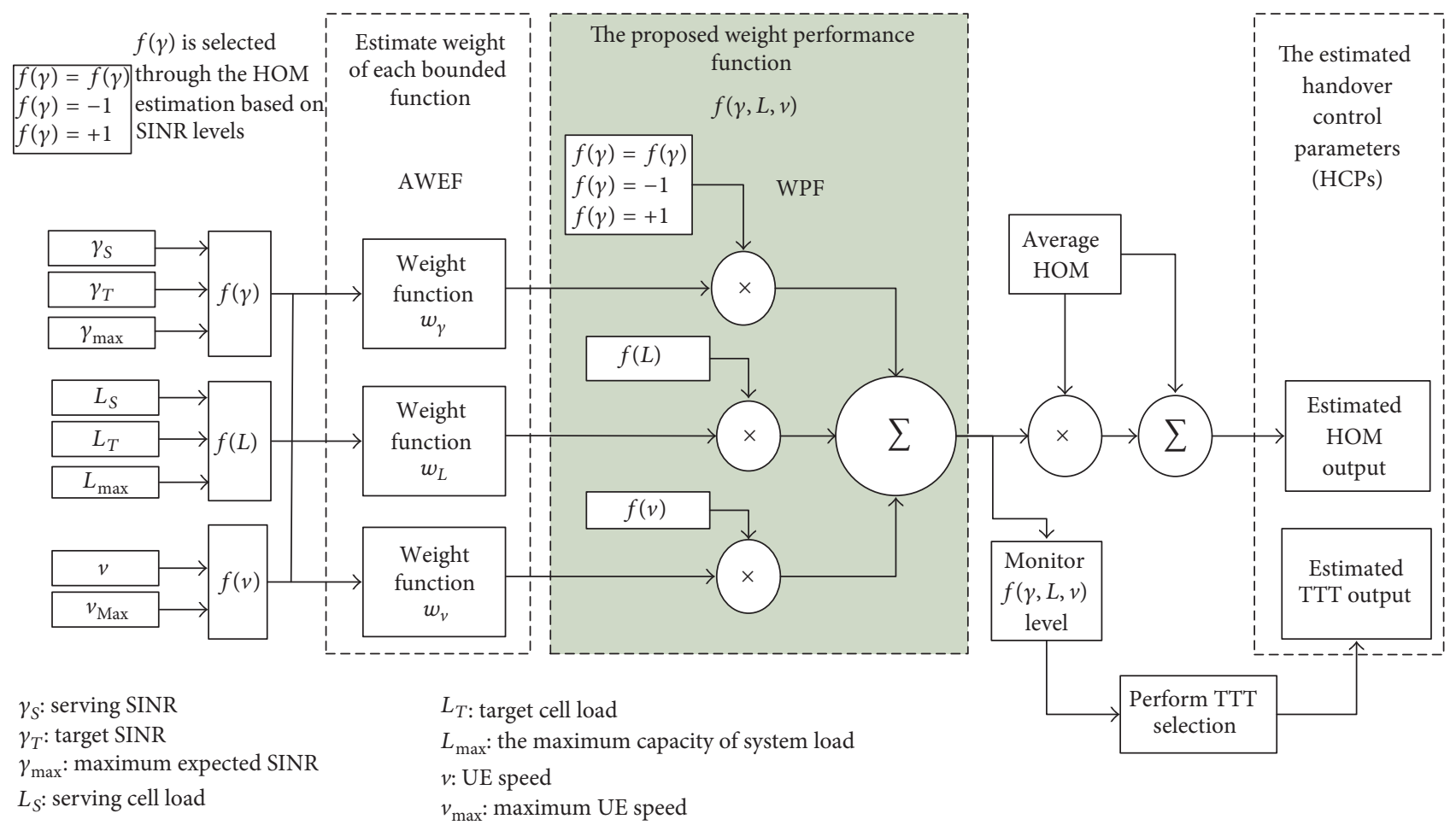

FIGURE 3: The proposed NHPO-WPF algorithm for estimating HCPs values.

the same. Additionally, the path loss differences between these two CCs are insignificant compared to the path loss differences between $\mathrm{CC} 1$ and $\mathrm{CC} 2$ that result from CADS3. Therefore, CC-CADS is expected to provide sufficient coverage over both CCs and will support better mobility everywhere around the serving eNB, which leads to enhanced spectral efficiency and a reduced outage probability for the UE.

3.2. Proposed Optimal Handover Parameter Optimization Algorithm. In this paper, a NHPO-WPF algorithm is proposed to automatically estimate the appropriate HCPs values, as described in Figure 3. This algorithm performs optimization based on a new proposed Weight Performance
Function (WPF) $\left(f_{\mathrm{WPF}}(\gamma, L, v)\right)$. The WPF is evaluated based on three bounded functions $f(\gamma), f(L)$, and $f(v)$, which are evaluated as functions of UE's $\operatorname{SINR}(\gamma)$, traffic load $(L)$, and UE's velocity $(v)$. The weight of each bounded function is taken into account to estimate accurate HCPs values. Thus, the NHPO-WPF algorithm will estimate the suitable HCPs values, such as the HOM level and TTT interval for each UE independently. The proposed WPF is formulated as follows:

$$
f_{\mathrm{WPF}}(\gamma, L, v)=\omega_{\mathrm{sinr}} \cdot f(\gamma)+\omega_{L} \cdot f(L)+\omega_{v} \cdot f(v),
$$

where $\omega_{\text {sinr }}, \omega_{L}$, and $\omega_{v}$ represent the weights of $f(\gamma), f(L)$, and $f(v)$, respectively. 
The weight of each bounded function $\left(\omega_{\text {sinr }}, \omega_{L}\right.$, and $\left.\omega_{v}\right)$ is automatically determined by an automatic proposed weight estimator function (AWF), which is formulated as

$$
\omega_{x}=\frac{1-f(x)}{\sum_{i=1}^{F}\left(1-f\left(x_{i}\right)\right)},
$$

where $\omega_{x}$ represents the weight of function $f(x)$, which can be $f(\gamma), f(L)$, or $f(v), F$ denotes the optimizing parameters factor, which represents the total number of parameters that are considered for optimizing HCPs (this is set to 3 because only three factors are considered $(\gamma, L$, and $v)$ ), and $f\left(x_{i}\right)$ is a function of $x_{i}$, whereas $x_{1}, x_{2}$, and $x_{3}$ denote $\gamma, L$, and $v$, respectively.

$f(\gamma)$ is a function of the SINR, which is expressed by

$$
f(\gamma)=\frac{\gamma_{T}-\gamma_{S}}{\gamma_{\max }}
$$

where $\gamma_{S}$ and $\gamma_{T}$ represent the SINRs over the serving PCC and the selected target CCs, respectively, and $\gamma_{\max }$ is the maximum expected SINR level measured at the UE, which is assumed to be $30 \mathrm{~dB}$. by

$f(L)$ is a function of the traffic loads, which is expressed

$$
f(L)=\frac{L_{T}-L_{S}}{L_{\max }},
$$

where $L_{T}$ and $L_{S}$ represent the occupant target and serving traffic loads, respectively, and $L_{\max }$ represents the maximum load capacity of the system.

$f(v)$ is a bounded function that is evaluated as a function of the UE's speed $v$. It is expressed by

$$
f(v)=2 \cdot \log _{2}\left(1+\frac{v}{v_{\max }}\right)-1,
$$

where $v$ represents the UE's velocity and $v_{\max }$ represents the maximum expected velocity of the UE. It is assumed roughly to be $140 \mathrm{~km}$ for theoretical investigation. In the actual system, it can also be assumed based on the actual environment (i.e., urban, suburban area).

The estimated value of $f_{\mathrm{WPF}}(\gamma, L, v)$ is used to estimate the HOM level and to select the suitable TTT interval for each UE independently as illustrated in Figure 3. The HOM level is estimated by multiplying $f_{\mathrm{WPF}}(\gamma, L, v)$ by the average $\mathrm{HOM}$ level $\left(M_{\text {Avg }}\right)$, and the result is combined with $M_{\text {Avg }}$, which is evaluated by

$$
M_{\mathrm{Avg}}=\frac{\left(M_{\max }-M_{\min }\right)}{2}
$$

where $M_{\max }$ and $M_{\min }$ denote the maximum and minimum handover margin, which are set to $10 \mathrm{~dB}$ and $0 \mathrm{~dB}$, respectively.

Similar to the HOM, TTT intervals are estimated dynamically through the computed $f_{\mathrm{WPF}}$ values. This dynamic update of the TTT intervals provides a more accurate determination of the TTT as compared to the TTT steps defined in the 3GPP standard. The update in TTT is denoted as $\Delta T$, which is estimated by the following model:

$$
\Delta T= \begin{cases}Z 1 & \text { if } T_{\min }<T<T_{\max } \\ Z 2 & \text { if } T=T_{\min } \\ Z 3 & \text { if } T=T_{\max },\end{cases}
$$

where $Z 1, Z 2$, and $Z 3$ are represented by (8), (9), and (10), respectively:

$$
\begin{aligned}
& Z 1= \begin{cases}T-\rho & \text { if } f_{\mathrm{WPF}} \leq f_{\mathrm{WPF}}+\mathbb{Q} \\
T+\rho & \text { if } f_{\mathrm{WPF}} \geq f_{\mathrm{WPF}}+\mathbb{Q}\end{cases} \\
& Z 2= \begin{cases}T & \text { if } f_{\mathrm{WPF}} \leq f_{\mathrm{WPF}}+\mathbb{Q} \\
T+\rho & \text { if } f_{\mathrm{WPF}} \geq f_{\mathrm{WPF}}+\mathbb{Q}\end{cases} \\
& Z 3= \begin{cases}T-\rho & \text { if } f_{\mathrm{WPF}} \leq f_{\mathrm{WPF}}+\mathbb{Q} \\
T & \text { if } f_{\mathrm{WPF}} \geq f_{\mathrm{WPF}}+\mathbb{Q},\end{cases}
\end{aligned}
$$

where $\rho$ and $Q$ represent the optimization interval and step level, respectively.

The constants, $\rho$ and $\mathcal{Q}$, are meant to adjust the resolution in which the TTT intervals are updated. If these constants are selected to be small, higher resolution of TTT is achieved. However, too high TTT resolution may impose high computational complexity and delays to the system. Thus, for simplicity, the values of $\rho$ and $Q$ are selected to be $0.04 \mathrm{~s}$ and 0.1 , respectively, throughout all the simulations. Furthermore, it can be noticed that when the update value is saturated at $T_{\max }$ or $T_{\min }$, then no further update is considered. $T_{\max }$ or $T_{\text {min }}$ is determined from the $3 \mathrm{GPP}$ recommendations as $0.0 \mathrm{~s}$ and $5.12 \mathrm{~s}$, respectively.

The initial values of HOM and TTT for all the implemented HPO algorithms are assumed to be $2 \mathrm{~dB}$ and 100 milliseconds, respectively.

For more simplicity, the proposed NHPO-WPF algorithm is simplified and summarized in Table 2. Meanwhile, it is compared with some of the most related algorithms selected from the literature. In this comparison, the significant factors that are used to optimize handover control parameters are presented. These factors can be briefly defined as follows.

Optimization Factors. Optimization factors are the influence elements in the algorithm that are used to optimize handover control parameters.

Optimized HCPs. They are the handover control parameters that are considered to be optimized (estimated) automatically based on certain condition.

Initial HCPs Values. They are the initial handover control parameters values that are introduced at the initial setup of the system. 
TABLE 2: Comparison the proposed NHPO-WPF algorithm with the most HPO related algorithms.

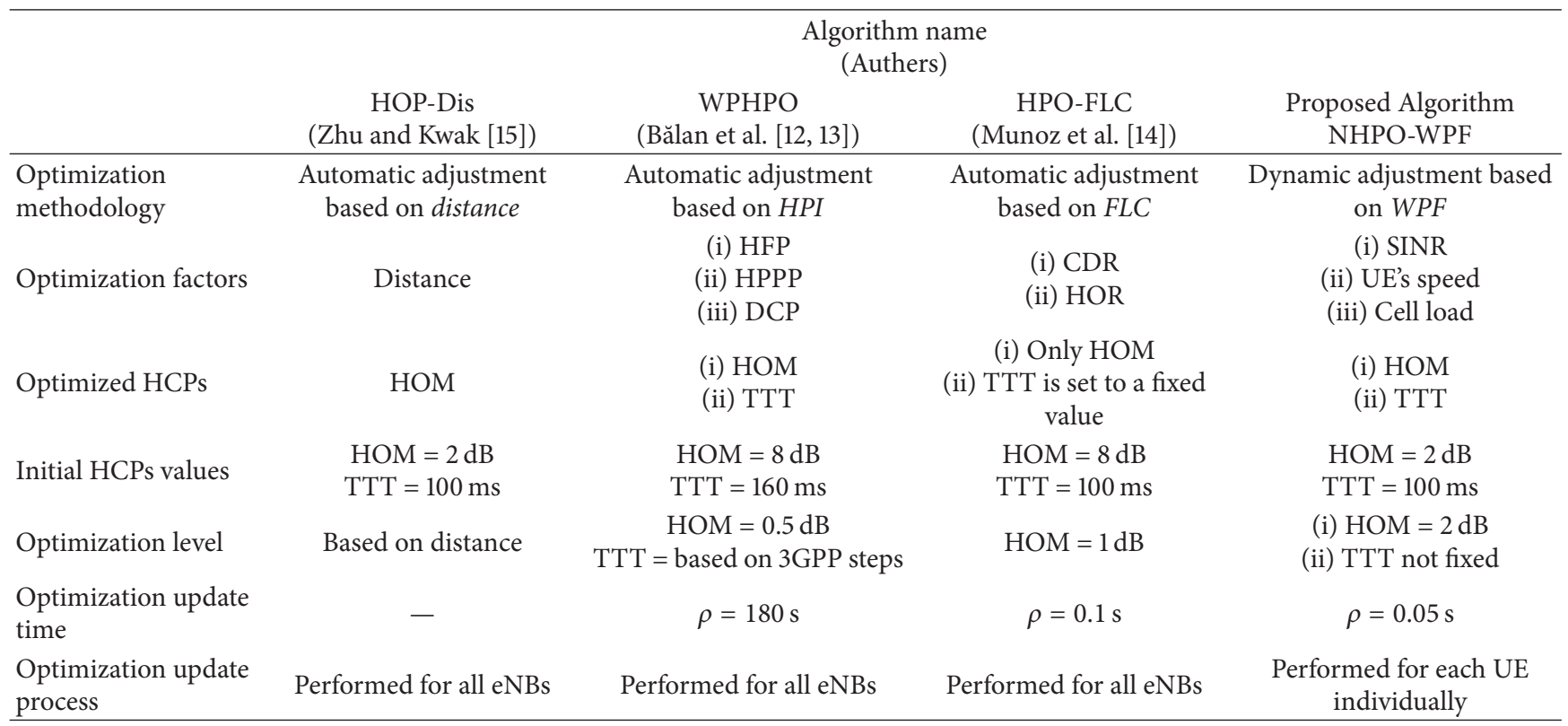

Optimization Level. It is the increment or decrement level in the handover control parameters.

Optimization Update Time. It is the duration that is separated between two optimization processes.

Optimization Update Process. It is the level of optimization over the system; for example, the optimization is performed for one UE, sector, eNB, or overall the system.

\section{Simulation Model}

4.1. System Layout Model. The LTE-Advanced system can be modeled as shown in Figure 4 and is built based on 3GPP specifications that were introduced in $[16,17]$. The network consists of 61 macrohexagonal cell layout models, which are built with an intersite distance of $500 \mathrm{~m}$ for each cell. Every hexagonal cell contains one eNB at its center, and each cell consists of three sectors with two aggregated CCs in each sector. Therefore, the network contains 61 cells, which are equivalent to 183 sectors. The transmission powers from the eNBs in the CCs are assumed to be the same. However, the six eNBs that are located in the first tier are considered to be the stations that cause interference to the UE during the simulation time at any position $x$. The movement of all the UEs is considered to occur only in the first 37 hexagonal cells. Thus, when the UE moves from the serving to the target eNBs, it should be surrounded by six eNBs. These six eNBs are considered to be the stations that cause the interference for the UE.

The Frequency Reuse Factor (FRF) is assumed to be one, 200 UEs are generated randomly in the serving cell, and the UEs in the target eNBs are generated and removed randomly.

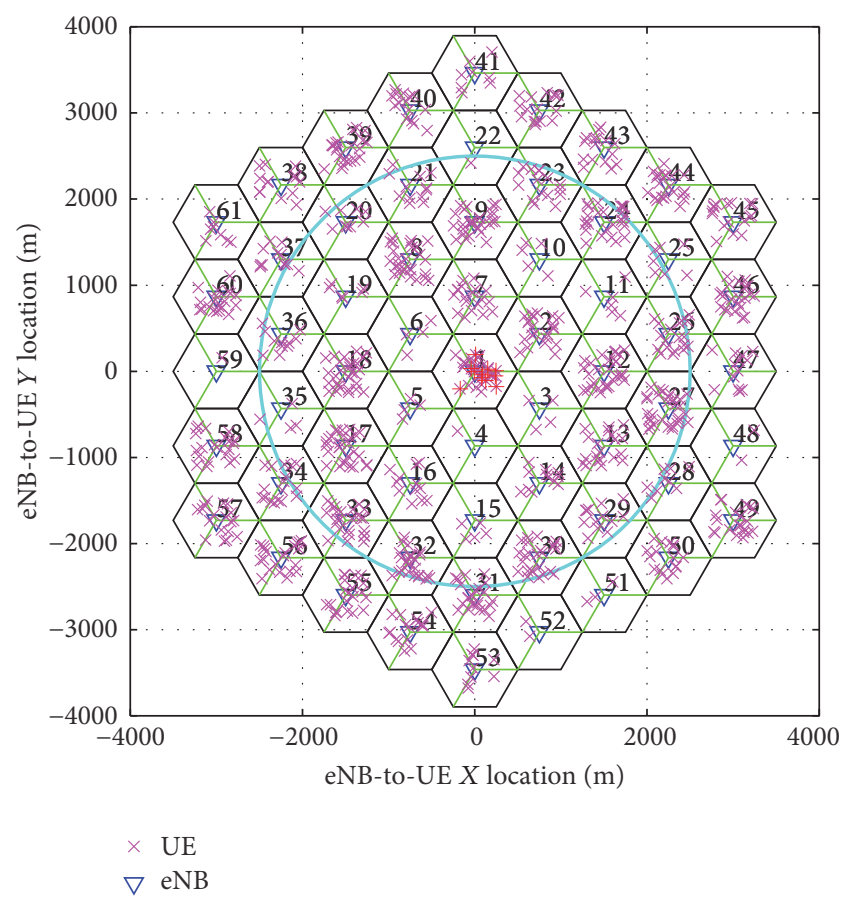

FIGURE 4: LTE-Advanced system model with 61 hexagonal cells, each of which consists of three sectors.

The random generation and removal of UEs in the target eNBs are intended to mimic the random generation of traffic in the simulation. The UEs are generated at random uniform positions in the cells, and each UE moves randomly at a fixed speed throughout the simulation, which contains ten different mobile speeds. The speeds range from typical vehicle 


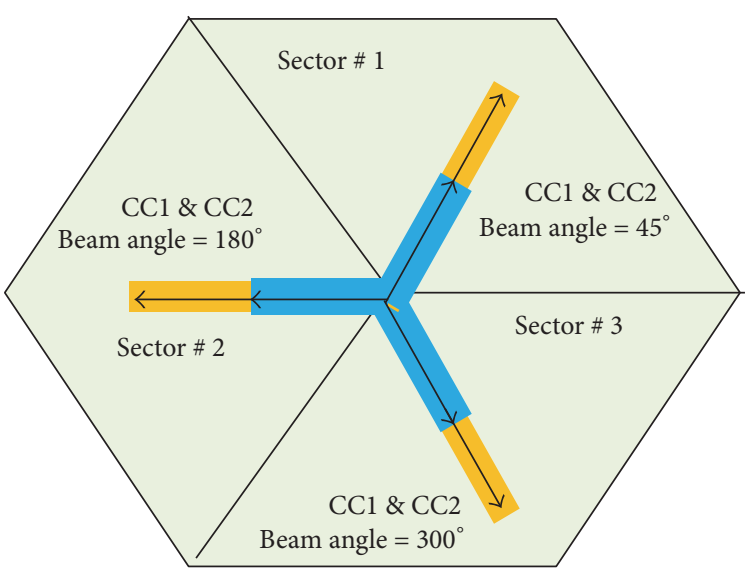

(a) CADS-1 and CADS-2

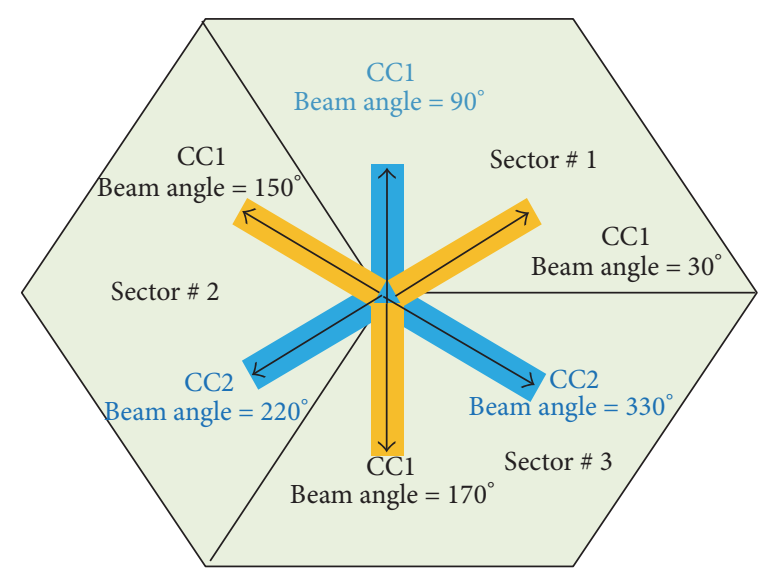

(b) CC-CADS and CADS-3

FIgURE 5: Beam directions of CC1 and CC2 based on different CADSs.

speeds in urban areas ( $40 \mathrm{~km} /$ hour) to a high train speed scenario $(140 \mathrm{~km} /$ hour$)$. The Adaptive Modulation and Coding (AMC) scheme is considered based on the sets of modulation schemes (MS) and Coding Rate (CR) that were introduced in [18-20]. In addition, to achieve accuracy in the high performance evaluation, detailed models for the handover procedure for LTE, the RLF detection, the reestablishment procedure, and the Non-Access Stratum (NAS) recovery procedure are considered in the simulation. The essential parameters that are used in the simulation are listed in Table 3. These parameters are taken based on LTE-Advanced system profile that was defined by $3 \mathrm{GPP}$ specifications [16-22].

4.2. Configuration of Carrier Aggregation Deployment Scenarios. Three CA deployment scenarios are considered and compared with CC-CADS. In CADS-1, the operating frequencies for $\mathrm{CC} 1$ and $\mathrm{CC} 2$ are assumed to be $2 \mathrm{GHz}$ and $2.023 \mathrm{GHz}$, respectively, and the beams of both CCs are directed in the same directions as shown in Figure 5(a). In CADS-2, the operating frequencies for $\mathrm{CC} 1$ and $\mathrm{CC} 2$ are assumed to be $2 \mathrm{GHz}$ and $3.5 \mathrm{GHz}$, respectively, and the beams of both CCs are directed in the same directions Figure 5(a). In CADS-3, the operating frequencies for CC1 and $\mathrm{CC} 2$ are assumed to be $2 \mathrm{GHz}$ and $3.5 \mathrm{GHz}$, respectively, and the beam of each CC is directed toward the cell boundary of the other CC. In CC-CADS, the proposed operating frequencies for $\mathrm{CC} 1$ and $\mathrm{CC} 2$ are assumed to be $2 \mathrm{GHz}$ and $2.023 \mathrm{GHz}$, respectively, and the beam of each CC is directed toward the cell boundary of the other CC. All the operating frequencies are assumed based on the agreed band scenarios for the Rel. 12 timeframe [17]. However, both CCs in CCCADS are expected to provide sufficient coverage, and both CCs can support mobility.

In CADS-3 and CC-CADS, the beam of each CC is directed in a different direction, and each carrier is pointed toward a different flat side of the hexagonal cell for all threesector sites as shown in Figure 5(b). Thus, the main beam
TABLE 3: Simulation parameters [16-22].

\begin{tabular}{|c|c|}
\hline Parameter & Assumption \\
\hline Cellular layout & $\begin{array}{l}\text { Hexagonal grid, } 61 \text { hexagonal } \\
\text { cells, } 3 \text { sectors per cell, } 2 \text { CCs } \\
\text { per sector }\end{array}$ \\
\hline $\begin{array}{l}\text { Minimum distance between UE } \\
\text { and eNB }\end{array}$ & $\geq 35$ meters \\
\hline Total eNB TX power & $46 \mathrm{dBm}$ per $\mathrm{CC}$ \\
\hline Shadowing standard deviation & $8 \mathrm{~dB}$ \\
\hline White noise power density $\left(N_{t}\right)$ & $-174 \mathrm{dBm} / \mathrm{Hz}$ \\
\hline eNBs noise figure & $5 \mathrm{~dB}$ \\
\hline Thermal noise power & $N_{P}=N_{t}+10 \log \left(\mathrm{BW} \times 10^{6}\right) \mathrm{dB}$ \\
\hline UE noise figure & $9 \mathrm{~dB}$ \\
\hline Operation carrier bandwidth & $\begin{array}{c}20 \mathrm{MHz} \text { for each, carrier PCC } \\
\text { and SCC }\end{array}$ \\
\hline Total system bandwidth & $40 \mathrm{MHz}(2 \mathrm{CCs} \times 20 \mathrm{MHz})$ \\
\hline Number of PRBs/CCs & $100 \mathrm{PRB} / \mathrm{CC}$ \\
\hline Number subcarriers/RBs & 12 subcarriers per $\mathrm{RB}$ \\
\hline $\begin{array}{l}\text { Number of OFDM symbols per } \\
\text { subframe }\end{array}$ & 7 \\
\hline Subcarrier spacing & $15 \mathrm{kHz}$ \\
\hline Resource block bandwidth & $180 \mathrm{kHz}$ \\
\hline Q_rxlevmin & $-101.5 \mathrm{~dB}$ \\
\hline Measurement interval & $50 \mathrm{~ms}$ for PCC and SCC \\
\hline Time-to-Trigger (TTT) range & 0 to $5120 \mathrm{~ms}$ \\
\hline $\mathrm{HO}$ margin & Selected adaptively $[\mathrm{dB}]$ \\
\hline Each $X 2$-interface delay & $10 \mathrm{~ms}$ \\
\hline Each eNB process delay & $10 \mathrm{~ms}$ \\
\hline T310 & $10 \mathrm{~s}$ \\
\hline$T_{\text {critical }}$ & 2 seconds \\
\hline
\end{tabular}

of CC2 is directed in a different direction than the main beam of $\mathrm{CC} 1$, and the beam of $\mathrm{CC} 2$ is directed toward the 


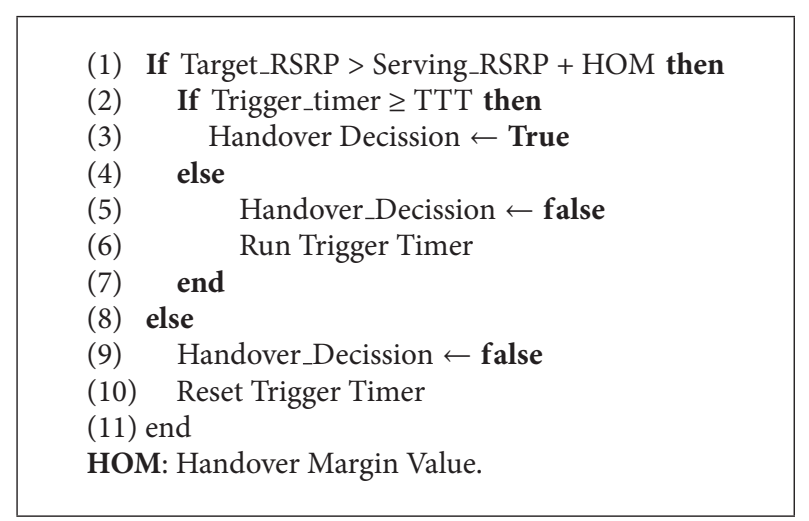

Algorithm 1: Handover decision algorithm.

cell boundary of CC1. Therefore, the beams of CC1 in sectors 1,2 , and 3 are aimed at beam angles of $30^{\circ}, 150^{\circ}$, and $270^{\circ}$, respectively, and the beams of CC2 in sectors 1,2 , and 3 are aimed at beam angles of $90^{\circ}, 210^{\circ}$, and $330^{\circ}$, respectively, as illustrated in Figure 5(b).

4.3. Simulation Scenario. In this paper, the RSRP is measured periodically during every measurement interval to evaluate the triggering Measurement Reports (MR) as performed in the real UE. The measurement is performed periodically for the PCCs and SCCs simultaneously from all neighboring eNBs based on the RSRP level. The best CC from each sector is then selected and ordered in a list based on the RSRP level. The cell that provides the best RSRP is always selected as the target cell candidate. After the target cell has been reported, the serving eNB will make a handover decision based on the best target cell. The serving eNB makes the handover decision based on the qualities of the serving RSRPs over the PCC and the quality of the selected target RSRPs. When the target RSRP is greater than the serving RSRP by the handover margin level during the TTT period, the serving eNB makes a handover decision and sends the handover request message to the target eNB. The handover decision can be expressed by Algorithm 1.

If the handover decision is true, the serving eNB prepares to perform the handover by sending a handover request message to the target eNB, and the UE will enter the handover procedure to establish a connection with the target eNB. The handover procedure is performed based on the handover procedure of the LTE-Advanced system as described in [16]. Once the target eNB receives the handover request message, it will start an admission control. If the admission control decision is true, the target $\mathrm{NB}$ will send a handover request acknowledge to the serving eNB, which in turn will begin the downlink (DL) allocation. Once the UE receives the RRCConnection-Reconfiguration message with the necessary parameters, it will begin to execute the handover to the target eNB.

The downlink RSRP is evaluated and updated periodically (whether the handover request has been sent or not) to detect the radio link connection's status. If a RLF is detected, the reestablishment request is sent to the target eNB to perform the Radio Resource Control (RRC) reestablishment procedure; the timer T310 (the maximum time allowed to recover a connection through the RRC reestablishment procedure) will be started, and cell reselection will be performed. Next, the UE attempts to find a suitable cell that can provide an RSRP greater than the minimum required receive level (Q_rxlevmin) in the cell. Once the UE finds a suitable cell, it will select that cell as the target cell; if the UE finds multiple suitable cells, the UE will select the best cell as the target cell. Once the target cell has been selected, the UE sends a reestablishment request message to the cell, and the RCC reestablishment procedure is performed. However, if the UE fails to find a suitable cell within the T310 period, the reestablishment procedure will fail, and the UE proceeds to the NAS recovery procedure. If the RRC reestablishment attempt fails, the UE will attempt to perform the NAS recovery procedure to recover the connection. The UE will continue with the attempt to find a suitable cell after the timer $T 310$ has expired; once it finds a suitable cell, it will perform the NAS recovery procedure on it. If the NAS recovery procedure fails, the UE will restart the search for a suitable cell. Once the UE finds a suitable cell, it will attempt to perform a NAS recovery procedure on the selected eNB again. The process of searching and performing the NAS recovery procedure will continue until the UE finds a suitable cell and successfully recovers the connection using the NAS recovery procedure. These recovery procedures are considered in the simulation to enhance the model and accurately evaluate the performance of the handover with the CA technique as performed in the real network. Moreover, all the failure events are counted together with the $U$-plane interruption time caused by these events.

4.4. Handover Scenarios. The introduction of the CA technique in mobile cellular systems creates an additional handover scenario, which leads to an increased handover rate. In LTE systems (Rel. 8 and 9), handover occurs between eNBs in different cells or between different sectors of the same cell. However, with the advent of the CA technique in LTEAdvanced systems, additional handovers occur between component carriers in the same sector, such as from F1 to F2 or from F2 to F1. Five handover scenarios can occur in an LTEAdvanced system based on CA technique: (i) interfrequency intrasector and intra-eNB handover, (ii) intrafrequency intersector and intra-eNB handover, (iii) interfrequency intersector and intra-eNB handover, (iv) intrafrequency inter-eNB handover, and (v) interfrequency inter-eNB handover [23]. All these handover scenarios are considered in this paper.

Intrafrequency means that the target and the serving carrier frequencies are the same, whereas interfrequency means that the target and serving carrier frequencies are different. Intrasector means that the target and serving sectors are the same and intersector means that the target and serving sectors are different. Intra-eNB means that the target and serving eNBs are the same, and inter-eNB means that the target and serving eNBs are different. All these handover scenarios are illustrated in Figure 6. 


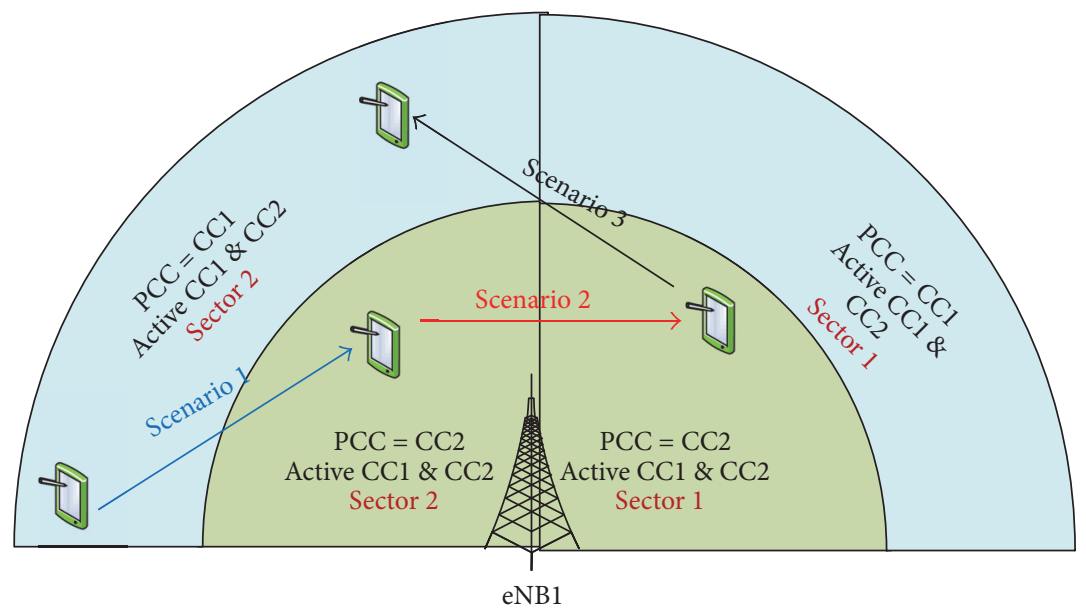

(a) Intra-eNB handover

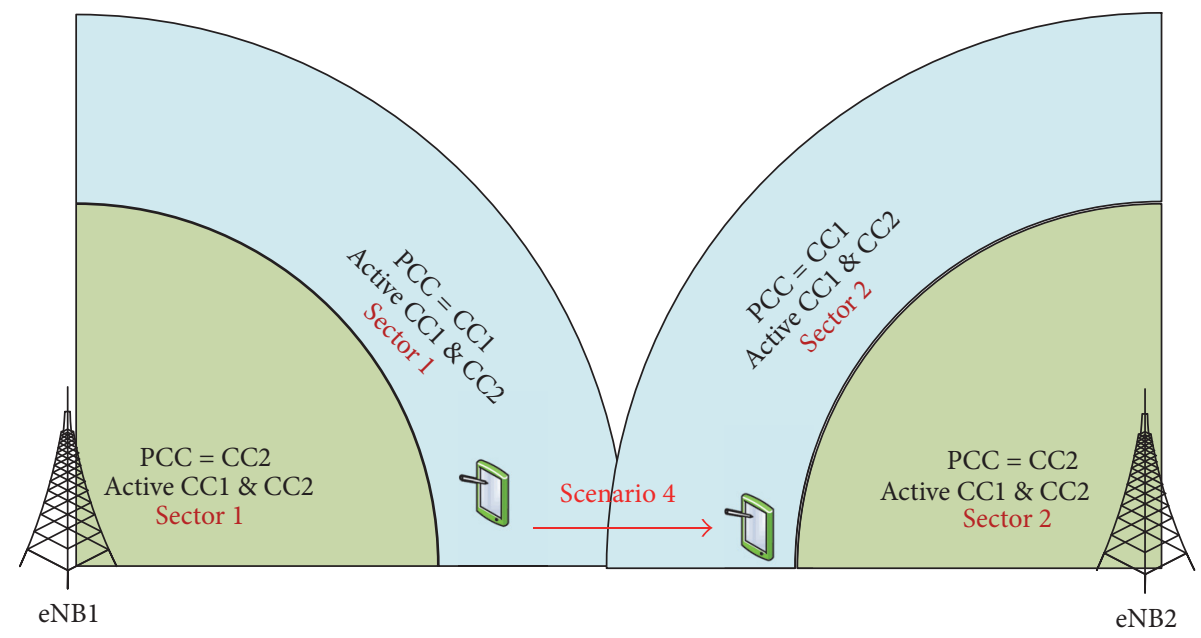

(b) Intrafrequency inter-eNB handover

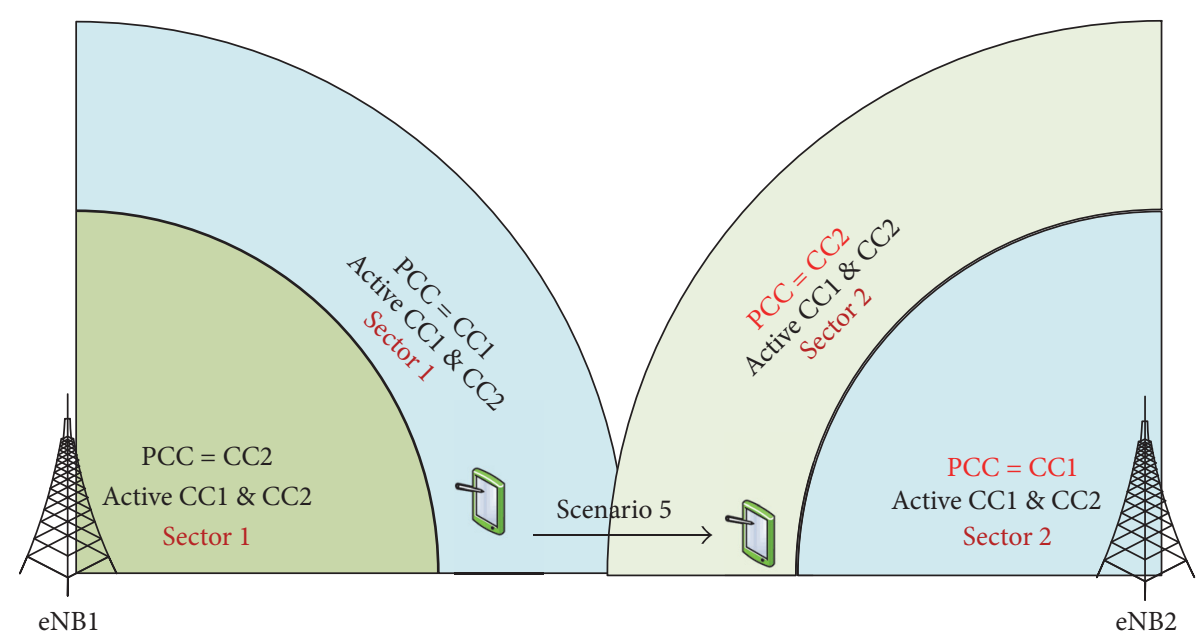

(c) Interfrequency inter-eNB handover

FIGURE 6: Frequency handover scenarios. 


\section{Evaluation of Handover Performance}

5.1. Downlink SINR Evaluation. This paper applies a macrocell propagation model that considers the path loss, shadowing, and Rayleigh fast fading effects. The propagation model can be formulated as [17]:

$$
\mathrm{PL}=58.8+37.6 \log _{10}(d)+21 \log _{10}\left(f_{c}\right)+\psi_{\mathrm{dB}}+\vartheta_{\mathrm{dB}},
$$

where $d$ represents the distance between the UE and the eNB in kilometers, $f_{c}$ is the operating carrier frequency in $\mathrm{MHz}$, $\psi_{\mathrm{dB}}$ is a log-normal shadowing in $\mathrm{dB}$, and $\vartheta_{\mathrm{dB}}$ represents the Rayleigh fast fading effect in $\mathrm{dB}$.

The transmitted signals in the DL transmission in an LTE-Advanced network based on the CA technique and an Orthogonal Frequency-Division Multiple Access (OFDMA) scheme are considered, where every eNB can serve each UE by $N_{\mathrm{sc}}^{\mathrm{UE}}$ subcarriers over $N_{\mathrm{CC}}^{\mathrm{UE}} \mathrm{CCs}$ assigned to each UE. This scenario means that each UE has the ability to receive data from multiple subcarriers $\left(N_{\mathrm{sc}}^{\mathrm{UE}}\right)$ over several CCs. The definition of the Physical Resource Block (PRB), which was introduced in [18-20], is considered in this paper. However, if the total number of subcarriers in a single CC is represented by $N_{\mathrm{sc}}^{\mathrm{CC}}$, the total transmission power $P_{\mathrm{TX}}$ of the eNB on each $\mathrm{CC}$ is distributed equally over all the subcarriers. Thus, the total transmission power of each subcarrier is expressed by [24]

$$
P_{\mathrm{TX}_{(m, k)}}=\frac{P_{\mathrm{TX}}}{N_{\mathrm{sC}}^{\mathrm{CC}}} .
$$

The transmitted power, $P_{\mathrm{TX}_{(m, k)}}$, over any subcarrier from any eNB in an LTE-Advanced system is assumed to be the same over any CC. Therefore, the useful received signal power $P_{\mathrm{RX}_{(m, k)}}$ at UE on subcarrier $k$ over $\mathrm{CC}_{m}$ in the DL transmission can be expressed by

$$
P_{\mathrm{RX}_{(m, k)}}=P_{\mathrm{TX}_{(m, k)}}+G_{\mathrm{TX}_{m}}+G_{\mathrm{RX}}-\mathrm{PL}_{m}(\mathrm{~dB}),
$$

where $P_{\mathrm{TX}_{(m, k)}}$ represents the transmitted signal power on subcarrier $k$ over $\mathrm{CC}_{m}$ in $\mathrm{dBm}, G_{\mathrm{TX}_{m}}$ represents the transmitter antenna gain over $\mathrm{CC}_{m}$ in $\mathrm{dB}, G_{\mathrm{RX}}$ represents the receiver antenna gain in $\mathrm{dB}$, and $\mathrm{PL}_{m}$ represents the path loss between $\mathrm{UE}$ and eNB over $\mathrm{CC}_{m}$ in $\mathrm{dB}$.

Only the interference signals received by the UE from the six neighboring eNBs located in the first tier that surrounds the served eNB are considered. The interference signals that are received from the eNBs located in the second tier will be neglected due to the weakness of these interference signals compared with those from the eNBs in the first tier. Thus, the interference signals received by the UE on subcarrier $k$ over $\mathrm{CC}_{m}$ from $H$ neighboring eNBs located in the first tier of the served eNB are expressed as

$$
I_{m, k}=\sum_{h=1}^{H} P_{\operatorname{int}_{(k, m h)}},
$$

where $P_{\text {int }_{\left(k, m \_h\right)}}$ represents the interference received signal power by the UE on subcarrier $k$ over $\mathrm{CC}_{m}$ from the neighboring eNB $h$.
Consequently, the SINR at the UE on subcarrier $k$ over $\mathrm{CC}_{m}$ is expressed by

$$
\operatorname{SINR}_{m, k}=\frac{P_{\mathrm{RX}_{(m, k)}}}{\sum_{h=1}^{H} P_{\text {int }_{(k, m, h)}}+P_{\mathrm{no}_{m, k}}},
$$

where $P_{\mathrm{no}_{m, k}}$ represents the noise power for the UE on subcarrier $k$ over $\mathrm{CC}_{m}$.

5.2. UE Bit Rate. Based on the 3GPP specifications introduced in $[18,25,26]$, one radio frame consists of ten subframes (i.e., one radio frame $=10 \mathrm{~ms}$ ), each subframe consists of two time slots, one time slot consists of $0.5 \mathrm{~ms}$ (i.e., 1 subframe $=1 \mathrm{~ms}$ ), and one time slot consists of 7 modulation symbols if a normal Cyclic Prefix (CP) length is used, in which the number of OFDMA symbols in each slot depends on the CP length and the configured subcarrier spacing. Each modulation symbol consists of 2, 4, or 6 bits if QPSK, 16-QAM, or 64-QAM is used as modulation scheme, respectively.

As explained in detail in $[18,26]$, the transmitted signal in each time slot is configured by one or several resource grids (RG), each RG consists of several PRBs $\left(N_{\mathrm{RB}}^{\mathrm{DL}}\right)$, each PRB consists of $N_{\mathrm{sc}}^{\mathrm{RB}}$ subcarriers, and each subcarrier is configured by $N_{\text {symb }}^{\mathrm{DL}}$ OFDMA symbols. The quantity of DL PRBs $N_{\mathrm{RB}}^{\mathrm{DL}}$ depends on the entire DL transmission bandwidth configured in the cell. Thus, a PRB consists of $N_{\text {symb }}^{\mathrm{DL}} \times N_{\mathrm{sc}}^{\mathrm{RB}}$ resource elements that correspond to one slot in the time domain and $180 \mathrm{kHz}$ in the frequency domain. Each modulation symbol carries $m_{\text {bit }}^{\text {symb }}$ bits, which depend on the modulation scheme that is selected. Consequently, the total number of bits in one time slot that consists of $N_{\text {symb }}^{\text {sc }}$ modulation symbols can be expressed by

$$
B_{\mathrm{bit}}^{\mathrm{sc}}=N_{\mathrm{symb}}^{\mathrm{sc}} m_{\mathrm{bit}}^{\mathrm{symb}} \text {. }
$$

Each PRB consists of $N_{\mathrm{sc}}^{\mathrm{RB}}$ subcarriers. Therefore, the total number of bits in one PRB $\left(B_{\mathrm{bit}}^{\mathrm{RB}}\right)$ can be given by

$$
B_{\text {bit }}^{\mathrm{RB}}=N_{\mathrm{sc}}^{\mathrm{RB}} N_{\mathrm{symb}}^{\mathrm{sc}} m_{\mathrm{bit}}^{\mathrm{symb}} .
$$

However, each $\mathrm{PRB}$ contains $N_{\mathrm{RS}}^{\mathrm{RB}}$ resource elements that are configured as reference symbols, which correspond to $N_{\mathrm{RS}}^{\mathrm{RB}}$ OFDM symbols in the time domain [26]. These, $N_{\mathrm{RS}}^{\mathrm{RB}}$, reference symbols allow the UE to estimate the channel condition. Therefore, the number of useful bits in one PRB can be given by

$$
B_{\mathrm{bit}}^{\mathrm{RB}}=N_{\mathrm{sc}}^{\mathrm{RB}}\left(N_{\text {symb }}^{\mathrm{sc}}-N_{\mathrm{RS}}^{\mathrm{RB}}\right) m_{\mathrm{bit}}^{\mathrm{symb}} .
$$

The total number of PRBs that can be assigned to each active $\mathrm{UE}\left(N_{\mathrm{RB}}^{\mathrm{UE}}\right)$ depends on the number of active UEs in the cell and the total available system bandwidth. The numbers of PRBs that can be assigned to each $\mathrm{UE}\left(N_{\mathrm{RB}}^{\mathrm{UE}}\right)$ can be expressed by

$$
N_{\mathrm{RB}}^{\mathrm{UE}}=\frac{N_{\mathrm{RB}}^{\text {Total DL }}}{N_{\mathrm{UEs}}^{\text {sys }}},
$$


where $N_{\mathrm{RB}}^{\text {Total DL }}$ represents the total number of available DL PRBs over the entire system bandwidth and $N_{\text {UEs }}^{\text {sys }}$ represents the total number of active UEs in the system. Consequently, the total number of useful bits that can be transmitted to each $\mathrm{UE} B_{\mathrm{bit}}^{\mathrm{UE}}$ can be expressed by

$$
B_{\mathrm{bit}}^{\mathrm{UE}}=N_{\mathrm{RB}}^{\mathrm{UE}} N_{\mathrm{sc}}^{\mathrm{RB}} N_{\mathrm{symb}}^{\mathrm{sc}} m_{\mathrm{bit}}^{\text {symb }} \text {. }
$$

The transmitted bits from the served eNB to the end UE include the code rate bits; therefore, the effect of the code rate, $E$, is considered in the evaluation. The total received UE throughput that can be correctly received from multiple CCs over the entire system bandwidth can be formulated by

$$
R_{\mathrm{bit}}^{\mathrm{UE}}=\frac{N_{\mathrm{RB}}^{\mathrm{UE}} N_{\mathrm{sc}}^{\mathrm{RB}}\left(\mathrm{N}_{\mathrm{symb}}^{\mathrm{sc}}-N_{\mathrm{RS}}^{\mathrm{RB}}\right) m_{\mathrm{bit}}^{\mathrm{symb}}}{T_{j}} E,
$$

where $T_{j}$ is the time over which the data bits are received for $\mathrm{UE}_{j}$.

5.3. Downlink Spectral Efficiency. The spectral efficiency can be represented mathematically by aggregating the total UE's throughput that is correctly received by the UE at a specific time and dividing by the total UE channel bandwidth. Therefore, the normalized spectral efficiency $\eta_{j}$ for $\mathrm{UE}_{j}$ can be expressed by [25]

$$
\eta_{j}=\frac{R_{\mathrm{bit}}^{\mathrm{UE}}}{T_{j} \omega_{\mathrm{BW}}^{\mathrm{UE}}}(\mathrm{bits} / \mathrm{sec} / \mathrm{Hz}),
$$

where $R_{\mathrm{bit}}^{\mathrm{UE}}$ denotes the number of correctly received bits for $\mathrm{UE}_{j}$ in a system and $\omega_{\mathrm{BW}}^{\mathrm{UE}}$ represents the UE's channel bandwidth, which can be calculated by multiplying the number of PRBs assigned to $\mathrm{UE}_{j}, N_{\mathrm{RB}}^{\mathrm{UE}}$, by the PRB's bandwidth $\left(B_{\mathrm{RB}}\right)$ and can be expressed by

$$
\omega_{\mathrm{BW}}^{\mathrm{UE}}=N_{\mathrm{RB}}^{\mathrm{UE}} B_{\mathrm{RB}} .
$$

Consequently, from (21) and (22), the UE's spectral efficiency based on a single component carrier can be expressed by

$$
\eta_{j}=\frac{N_{\mathrm{RB}}^{\mathrm{UE}} N_{\mathrm{sc}}^{\mathrm{RB}}\left(N_{\mathrm{symb}}^{\mathrm{sc}}-N_{\mathrm{RS}}^{\mathrm{RB}}\right) m_{\mathrm{bit}}^{\mathrm{symb}}}{T_{j} \omega_{\mathrm{BW}}^{\mathrm{UE}}} E(\mathrm{bps} / \mathrm{Hz}) .
$$

Because this study considers the CA technique based on $U$ component carries, the total UE's spectral efficiency can be formulated based on (21) as

$$
\eta_{j}=\sum_{m=1}^{U} \frac{N_{\mathrm{RB}}^{\mathrm{UE}}\left(\mathrm{CC}_{m}\right) N_{\mathrm{sc}}^{\mathrm{RB}}\left(N_{\mathrm{symb}}^{\mathrm{sc}}-N_{\mathrm{RS}}^{\mathrm{RB}}\right) m_{\mathrm{bit}}^{\mathrm{symb}}}{T_{j} \omega_{\mathrm{BW}}^{\mathrm{UE}}} E(\mathrm{bps} / \mathrm{Hz}) .
$$

5.4. Cell Edge UE's Spectral Efficiency. The cell edge spectral efficiency is an important measurement performance metric that is used to evaluate the throughput at the cell boundary in UE mobility studies of cellular communication systems. Because the proposed CA deployment scenario and CADS3 are scenarios that can contribute to enhancing the cell edge throughput, the cell edge UE's spectral efficiency will be evaluated to identify the enhancements that can be achieved in each scenario. The cell edge throughput will be evaluated to assess the enhancement that can be achieved at the cell boundary using the proposed CC-CADS compared to standard CADSs. The cell edge UE's spectral efficiency can be defined as the 5 th percentile of the Cumulative Distribution Function (CDF) of the normalized UE's spectral efficiency [23], which is defined as the average UE throughput over an appointed period divided by the channel bandwidth as measured in bit/s/Hz. Therefore, the cell edge UE's spectral efficiency is a measure of the perceived "quality of service" for the $5 \%$ of UEs with the lowest UE throughput.

5.5. Handover Probability. The handover probability (HOP) is the likelihood of switching the radio link connection for the served UE from the source to the target cells during active mode operation [27]. In other words, HOP is the probability of handing over the served UE from the serving to the target cells once the serving signal quality is becoming worse than the target signal strength by a HOM level. HOP is a significant performance indicator that is used to measure system performance and can be represented by

$$
P_{\mathrm{HO}}=P_{r}\left[\beta_{T}-\beta_{S} \geq M\right],
$$

where $\beta_{T}$ and $\beta_{S}$ represent the signal levels of the target and serving cells, respectively, and $M$ represents the HOM level. The handover probability can be translated into the average number of handovers per call over all the served UEs to increase the performance evaluation accuracy. The average handover probability rate is calculated in every simulation cycle over all the served UEs in the system. Thus, the average number of handovers per UE $\left(\overline{P_{\mathrm{HO}}}\right)$ can be expressed by

$$
\overline{P_{\mathrm{HO}}}=\frac{\sum_{j=1}^{N_{\mathrm{UEs}}^{\mathrm{sys}}} P_{\mathrm{HO}}(j)}{N_{\mathrm{UEs}}^{\mathrm{sys}}},
$$

where $N_{\text {UEs }}^{\text {sys }}$ represents the total number of served UEs over the system and $P_{\mathrm{HO}}(j)$ represents the handover probability for $\mathrm{UE}_{j}$.

5.6. Handover Ping-Pong Probability. HPPP is an important metric in studies of handover; it is used to measure the number of unnecessary handovers that are performed between two adjacent cells [27]. The handover will encounter the pingpong effect if UE-i leaves the serving eNB-A to the target eNB-B and is then handed back to the serving eNB-A in a period less than the critical interval $T_{\text {critical }}$ (the time required to measure the unnecessary handover between adjacent cells; it is assumed to be 2 seconds). When the handover takes place, the HPPP can be measured based on the following probability:

$$
P_{\text {HPPP }}=P\left[T_{\text {Interval }} \leq T_{\text {critical }}\right] \text {, }
$$

where $T_{\text {Interval }}$ represents the time interval between the UE leaving the serving eNB-A and being returned to the same eNB-A. Thus, $T_{\text {Interval }}$ can be expressed by

$$
T_{\text {Interval }}=T_{\text {Leave }}-T_{\text {handed back }} \text {, }
$$


where $T_{\text {Leave }}$ represents the time the UE leaves the serving eNB-A and $T_{\text {handed back }}$ represents the time the UE is handed back to the serving eNB-A. If the UE is handed back to the old serving eNB (eNB-A) and $T_{\text {Interval }}$ is less than $T_{\text {critical }}\left(T_{\text {Interval }}<T_{\text {critical }}\right)$, the handover is recorded as a ping-pong handover. The number of ping-pong handovers is recorded for each UE, and the average HPPP over all the served UEs is recorded in every simulation cycle $t$ to increase the accuracy of the performance evaluation. The average HPPP $\left(A_{\mathrm{HPPP}}\right)$ per UE during simulation cycle $t$ can be represented by

$$
A_{\mathrm{HPPP}}=\frac{N_{\mathrm{HPP}}^{\text {sys }}}{N_{\mathrm{RHP}}^{\text {sys }}}
$$

where $N_{\mathrm{HPP}}^{\text {sys }}$ represents the total number of handover pingpongs over all the system and $N_{\mathrm{RHP}}^{\text {sys }}$ is the total number of requested handovers, which is given by

$$
N_{\mathrm{RHP}}^{\text {sys }}=N_{\mathrm{SHP}}^{\text {sys }}+N_{\mathrm{FHP}}^{\text {sys }}
$$

where $N_{\text {SHP }}^{\text {sys }}$ and $N_{\text {No-HPP }}^{\text {sys }}$ are the numbers of successful and failed handovers. The number of successful handovers $\left(N_{\text {SHP }}^{\text {sys }}\right)$ includes the ping-pong $\left(N_{\text {HPP }}^{\text {sys }}\right)$ and non-ping-pong $\left(N_{\text {No-HPP }}^{\text {sys }}\right)$ handover numbers and is given by

$$
N_{\mathrm{SHP}}^{\text {sys }}=N_{\mathrm{HPP}}^{\text {sys }}+N_{\mathrm{No}-\mathrm{HPP}}^{\text {sys }} .
$$

5.7. Handover Failure Ratio. Handover failure normally occurs after the handover request has been sent to the target eNB [25]. Two cases can cause a handover failure: (i) lack of target resource availability and (ii) loss of coverage. In the former case, the handover failure occurs after the handover request is sent to the target $\mathrm{eNB}$ and the handover procedure is initiated but insufficient resources are available for the target eNB to complete the handover procedure. In the latter case, the handover failure occurs if the UE moves out of the coverage of the target eNB before the handover procedure is finalized. The total handover failure ratio $\left(N_{\mathrm{FHP}}^{\text {Totl }}\right)$ can be expressed as

$$
N_{\mathrm{FHP}}^{\text {Totl }}=\frac{N_{\mathrm{FHP}}^{\text {sys }}}{N_{\mathrm{FHP}}^{\text {sys }}+N_{\mathrm{SHP}}^{\text {sys }}} .
$$

5.8. Outage Probability. The outage probability $\left(P_{\text {out }}\right)$ of the cell can be defined as the percentage of area within the cell that does not meet its minimum power requirement $P_{\min }$, which can be defined as the probability that the instantaneously received $\operatorname{SINR}(\gamma)$ falls below a given threshold level, where the threshold level $\gamma_{\mathrm{Thr}}$ represents the minimum SINR level below which the performance becomes unacceptable. The outage probability for cellular mobile communication systems is represented mathematically as the probability that the instantaneously received $\operatorname{SINR}(\gamma)$ falls below the threshold level $\gamma_{\mathrm{Thr}}[28,29]$ and is normally represented as

$$
P_{\text {out }}=P\left[\gamma<\gamma_{\mathrm{Thr}}\right]=1-P\left[\gamma>\gamma_{\mathrm{Thr}}\right] .
$$

In this simulation, the outage probability is recorded when the serving SINR of $\mathrm{UE}_{j}$ during simulation cycle $t$ falls below

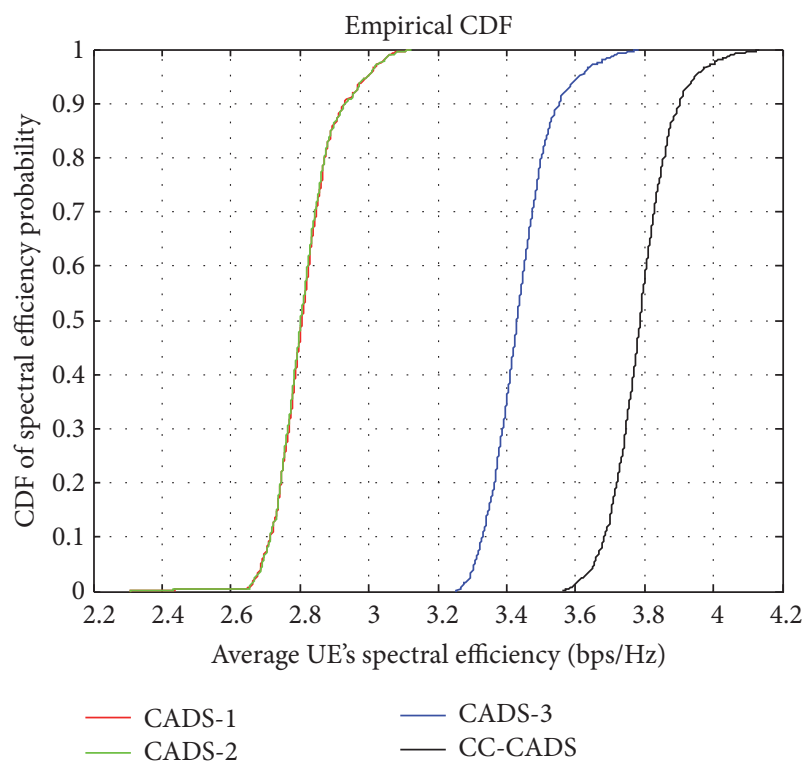

Figure 7: Average UE's spectral efficiencies with different CADSs.

a given threshold level, and the average outage probability for all UEs is evaluated during every simulation cycle to increase the accuracy of the results. From (34), the average outage probability can be simplified as

$$
P_{\text {out }}=\frac{\sum_{j=1}^{N} 1-P\left[\gamma_{j}>\gamma_{\mathrm{Thr}}\right]}{N_{\mathrm{UEs}}^{\text {sys }}} .
$$

\section{Results and Discussions}

In this section, the performance results of both proposed solutions will be presented and discussed. First, the achievable system performance results of CC-CADS will be presented and compared with those of three different CADSs. Then, the system performance results from the NHPOWPF algorithm based on CC-CADS will be presented and compared with the conventional HPO, WPHPO, and FLC algorithms.

6.1. Carrier Aggregation Deployment Scenario with Sufficient Coverage. This subsection presents the system performance results of CC-CADS and compares them with the results of three standard CADSs: CADS-1, CADS-2, and CADS-3. All the results presented in this subsection were simulated based on a conventional HPO algorithm with ten different mobile speeds. The results are presented in terms of the UE's spectral efficiency, the cell edge UE's spectral efficiency, and the outage probability. The main goal of CC-CADS is to enhance the spectral efficiency and reduce the outage probability.

Figures 7 and 8 show the average UE's spectral efficiencies and the average cell edge UE's spectral efficiencies, respectively. These results represent the average values over all UEs and all mobile speeds for four different CADSs. The results show that the CADS-1 and CADS-2 scenarios give the same spectral efficiency. These identical results are 


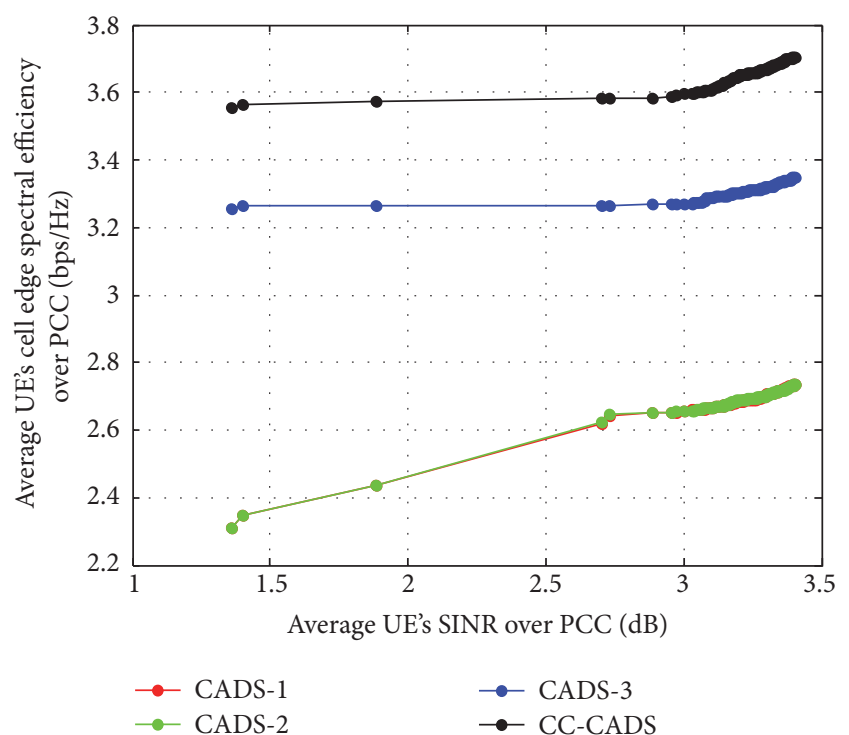

FIGURE 8: Average cell edge UE's spectral efficiency versus SINR for different CADSs.

due to the overlapping coverage that are provided by both scenarios, CADS-1 and CADS-2. The results in these two figures also illustrate that the third deployment scenario, CADS-3, provides better spectral efficiency than that are provided by CADS-1 and CADS-2. This enhancement is due to the different beams orientation of the aggregated CCs. Despite the fact that CADS-3 provides better enhancement, the path loss differences between the aggregated CCs are high, which leads to degrading the UE spectral efficiency. Thus, CC-CADS is proposed to further enhance UE spectral efficiency as well as the UE's outage probability. The results in Figures 7 and 8 show that the CC-CADS clearly provides a significant enhancement of the UE's spectral efficiency compared with CADS-1, CADS-2, and CADS-3 everywhere in the cell. The average UE's spectral efficiency achieved by CC-CADS is approximately 35\%, 35\%, and 10\% better than those achieved by CADS-1, CADS-2, and CADS-3, respectively, and the average cell edge UE's spectral efficiency achieved by CC-CADS is approximately $36 \%, 36 \%$, and $10 \%$ better than those achieved by CADS-1, CADS-2, and CADS3 , respectively. Thus, CC-CADS achieves better average UE's spectral efficiency than those achieved by CADS-1, CADS-2, and CADS-3 everywhere in the cell.

Figure 9 shows the average outage probabilities versus the different mobile speeds for four different CADSs. The results show that the outage probabilities that have resulted from the CADS-1 and CADS-2 are almost the same. The reason for that is similar to the reason that was illustrated in the previous paragraph. In additional to that, the UE's outage probability is mainly depending on the SINR performance over the PCC only. Since the aggregated CCs in CADS-1 are operating on a contiguous band and their beam orientations are the same, the SINR performances over all the aggregated CCs are mostly the same. Thus, the CC1 may be always configured as PCC. According to CADS-2, the aggregated $\mathrm{CCs}$ are operating on the noncontiguous band and their beam

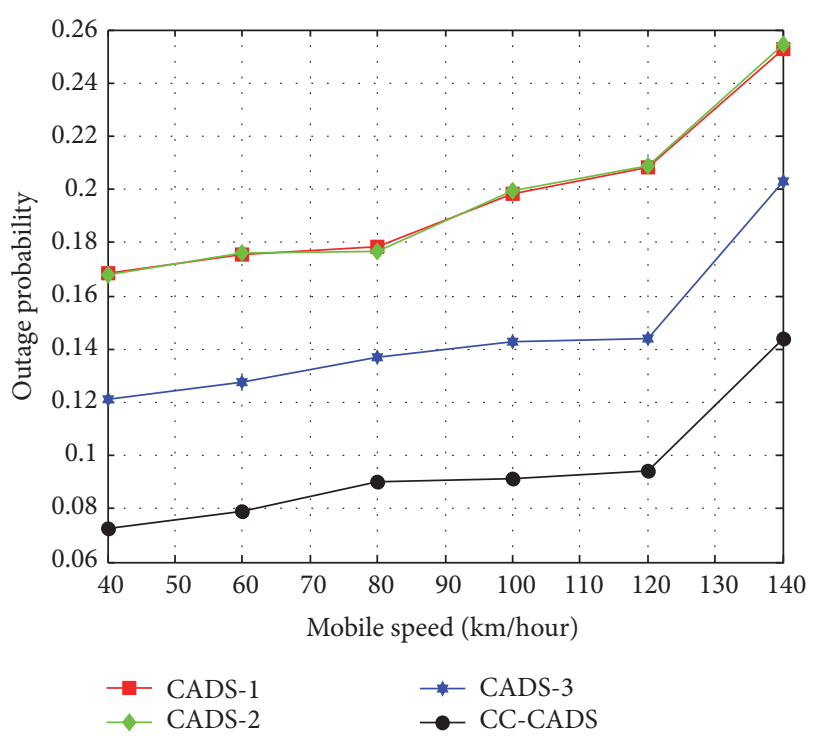

Figure 9: Outage probabilities with different CA deployment scenarios.

orientations are the same. Thus, the SINR performance over CC1 will be better than the SINR performance over CC2. Therefore, CC1 will be configured as PCC. Since the UE's outage probability always depends on the SINR performance over the PCC, thus, the resulted UE's outage probabilities from CADS-1 and CADS-2 will be almost the same. On the other hand, the UE's outage probability that resulted from CADS- 3 is less than that resulted from CADS-1 and CADS2. This may due to the different beam orientations for the aggregated CCs, which leads to enhancing the SINR at the CCs cell edge, in which it leads to enhancing the SINR over the PCC that is always being configured as the best CC providing highest SINR level. As a result of that, the UE's outage probability is enhanced. However, this provided enhancement by CADS-3 is not totally eliminating the UE's outage probability. Thus, CC-CADS is also proposed to further reduce UE's outage probability as much as possible as also presented in Figure 9. The results show that CCCADS provides a notable reduction in the outage probability compared to CADS-1, CADS-2, and CADS-3 in the different mobile speeds. The average outage probabilities achieved by CC-CADS over all the mobile speeds are $49.6 \%, 50 \%$, and $30.6 \%$ less than those of CADS-1, CADS-2, and CADS-3, respectively.

These enhancements in spectral efficiency and reductions in outage probability were achieved by the CC-CADS due to two main factors. The first factor is the beam direction of CC2, which is oriented toward the cell boundary of CC1. Therefore, the main beam direction of each CC was oriented toward the cell boundary of another CC, which increased the beam gains at the cell boundaries, thus providing the strongest serving RSRP level and in turn enhancing the UE's spectral efficiency everywhere around the serving eNB. Providing the strongest serving RSRP increased the serving SINR, which in turn led to reduced outage probabilities everywhere around the serving eNB. 
The second contributing factor is the operating frequencies for $\mathrm{CC} 1$ and $\mathrm{CC} 2$, which are assumed to operate in a contiguous band. The coverage areas provided by these two CCs are almost the same but have different beam directions. Thus, the path loss differences between these two CCs cannot be compared with those based on CADS-2 or CADS-3; the path loss that results from CC2 based on CADS- 3 will be higher than that from CC2 based on CC-CADS. CC-CADS provides a sufficient coverage area that is better than those provided by CADS-1, CADS-2, and CADS- 3 everywhere around the serving eNB, which leads to enhanced serving RSRP everywhere around the serving eNB. The enhancement of the serving RSRP led to an increase in the serving SINR to the UE, which in turn increased the UE's spectral efficiency and reduced the UE's outage probability. Thus, the CC-CADS provides better UE's spectral efficiency enhancement and outage probability reduction everywhere around the serving eNB than CADS-1, CADS-2, and CADS-3, as illustrated in Figures 7, 8, and 9, respectively.

6.2. Optimal Handover Parameter Optimization. In this subsection, the proposed NHPO-WPF, conventional HPO, FLC, and WPHPO algorithms are analyzed to investigate and validate their performance in the CA technique and to highlight the enhancements that are achieved by the proposed NHPOWPF algorithm as compared to the other algorithms. Firstly, an example of how the NHPO-WPF algorithm adapts the HCPs depending on the SINR, system load, and user speed is given. Then, the simulation results of these four HPO algorithms are presented and discussed based on CC-CADS. The results show the impact of different mobile speeds on the handover performance of proposed algorithm and the other three HPO algorithms. Because the proposed NHPO-WPF algorithm is intended to enhance handover performance, the simulation results are presented and discussed in terms of the average HOP, HPPP, and HFP. The average values are calculated over all active UEs and then over all the simulation time.

The handover control parameters estimated by the proposed NHPO-WPF algorithm during the simulation time are shown in Figure 10 based on the UE speeds of $120 \mathrm{~km} /$ hour only. The HOM and TTT are initialized at $2 \mathrm{~dB}$ and 100 milliseconds, respectively. The aim of this simulation is to highlight the comparative HOM and TTT values produced by the proposed algorithm at different UE speeds in contrast to the conventional and some of the literature algorithms. The HOM and TTT are computed as averages over all the UEs in this simulation. The results are presented for three-second time interval. It is clear that the conventional HPO algorithm shows decay in the HOM and the TTT at all UE speeds. This can be explained by noticing that HPO algorithms aim to reduce the RLF in the network; hence it tends to reduce the HCPs. On the other hand, the FLC algorithm provided higher HOM values as compared to the conventional HPO. However, the TTT profile produced by the FLC algorithm is in close matching to the conventional HPO. Similar to the conventional HPO algorithm, the UE speed influence on the HCPs values estimated by FLC algorithm is very minor.
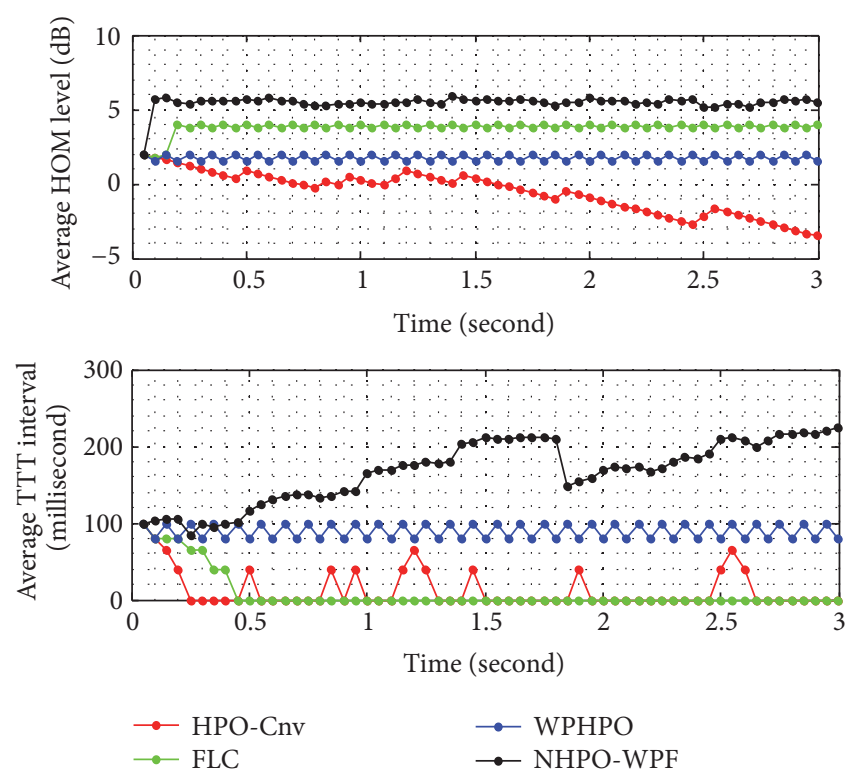

FIgURE 10: Average HCPs values versus time with UE speed of $120 \mathrm{~km} /$ hour.

The WPHPO algorithm estimates the HCPs parameters in the smaller range as compared to both HOP and FLC. This small estimation range may cause insufficient estimation of the HCPs values, particularly at high UE speeds. More importantly, the effect of UE speed on the performance of the WPHPO is also very minor.

As the proposed NHPO-WPF algorithm considers the UE performance metric as parameters to estimate the HCPs, it provided wider HCPs estimation range. This would provide appropriate HOM and TTT levels estimation at different UE speeds. This appropriate estimation may improve handover performance in general. Moreover, the effect of UE speeds influenced the estimation range of the proposed algorithm. This can be deduced by the comparison in Figure 10. This influence of the UE speeds is due to the consideration of UE speeds as estimation parameter in the NHPO-WPF algorithm.

Figure 11 shows the average handover probability versus mobile speed for the NHPO-WPF, conventional HPO, FLC, and WPHPO algorithms. The results show that the proposed NHPO-WPF algorithm provides a significant reduction of the average handover probability compared to the conventional HPO, FLC, and WPHPO algorithms for all the mobile speeds. The average HOPs that are achieved by the NHPOWPF algorithm are approximately 95, 98, and 90\% lower than those with the conventional HPO, FLC, and WPHPO algorithms, respectively. Because a high HOP leads to high HPPP and HFP, the reduction of HOP will lead to significant reductions in the HPPP and HFP, which will be discussed below.

Figures 12 and 13 show the average HPPPs for the NHPOWPF, conventional HPO, FLC, and WPHPO algorithms based on the different mobile speeds. HPPP may occur when a nonoptimal HPO algorithm is used to optimize the HCPs, which leads to estimating suboptimal HCPs values, in turn 


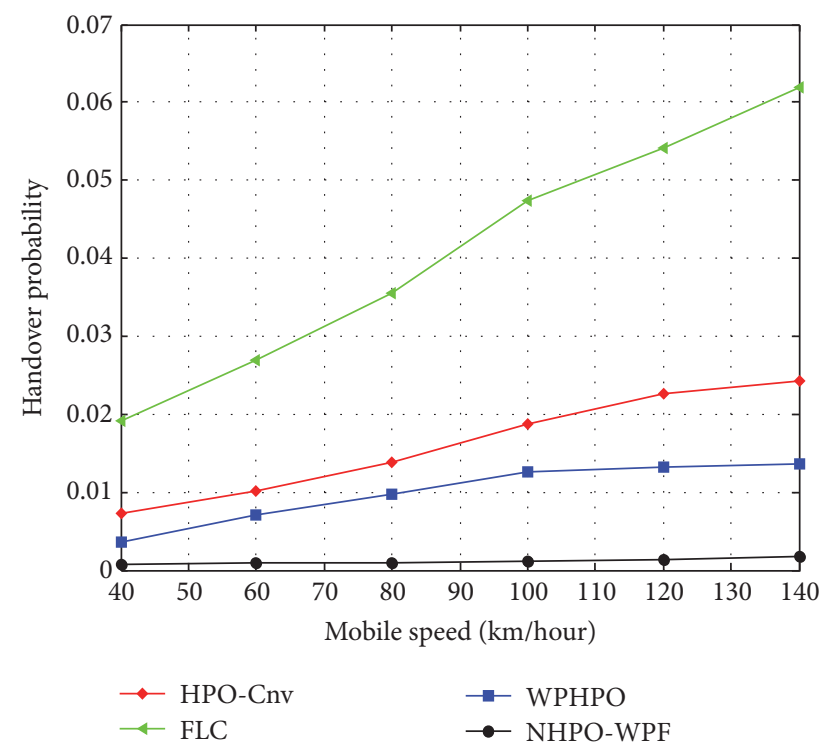

FIGURE 11: Average handover probability versus mobile speed with different handover parameter optimization algorithms.
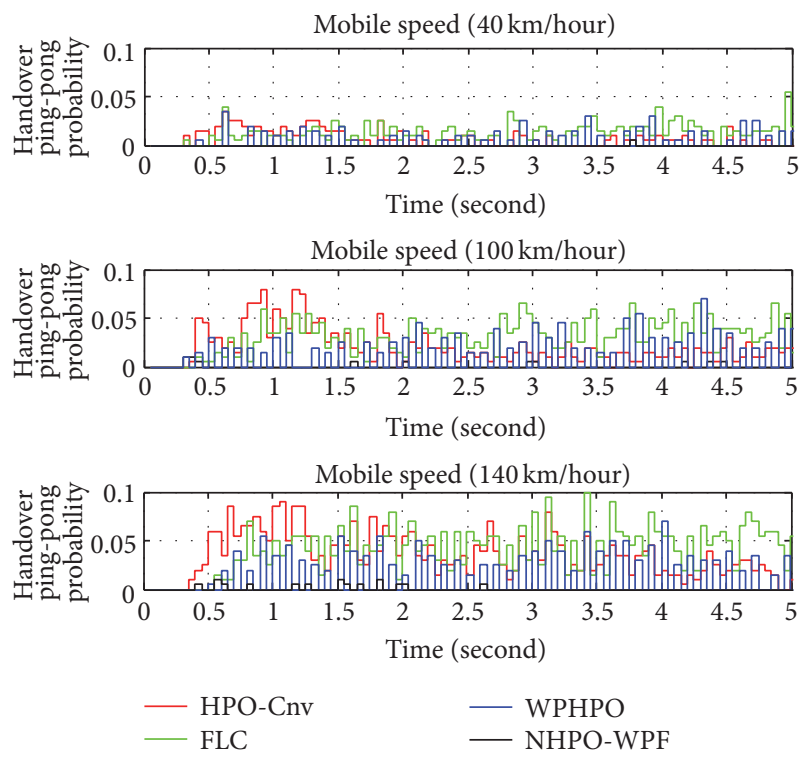

FIGURE 12: Handover ping-pong probability with different mobile speeds based on different HPO algorithms.

leading to an increase in the number of unnecessary handovers (HPPP effect), especially in high mobility speeds. This high HPPP effect increases the waste of network resources. The results shown in Figure 12 represent the average HPPP per UE based on the various mobile speeds (medium and high speeds), while the results shown in Figure 13 show the average HPPP over the entire simulation time for each mobile speed scenario independently. The results show that the proposed NHPO-WPF algorithm provides a lower HPPP than the conventional HPO, FLC, and WPHPO algorithms for all the considered mobile speeds scenarios; it achieves

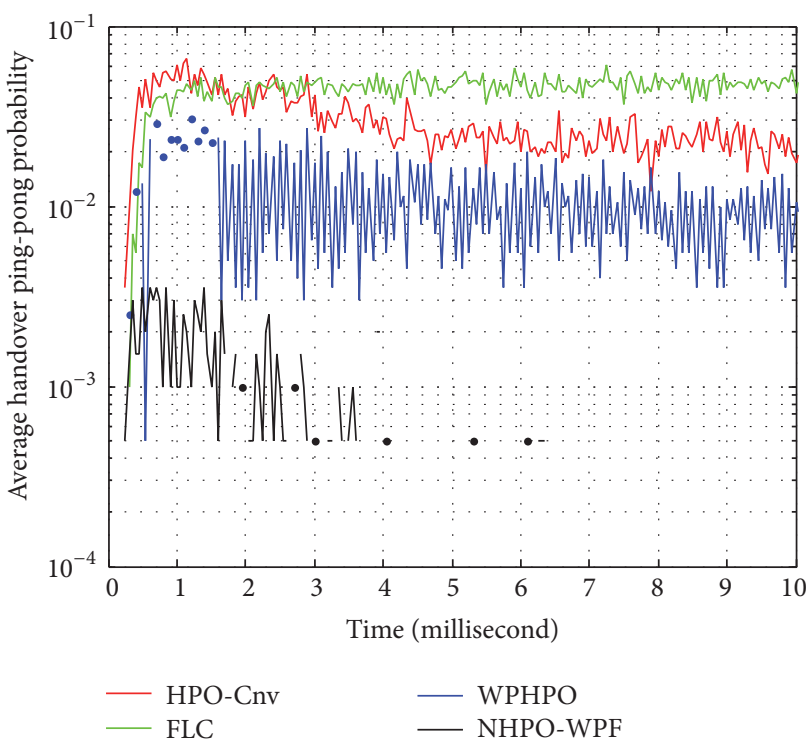

FIGURE 13: Average handover ping-pong probability over all mobile speeds versus time, based on the HPO algorithms.

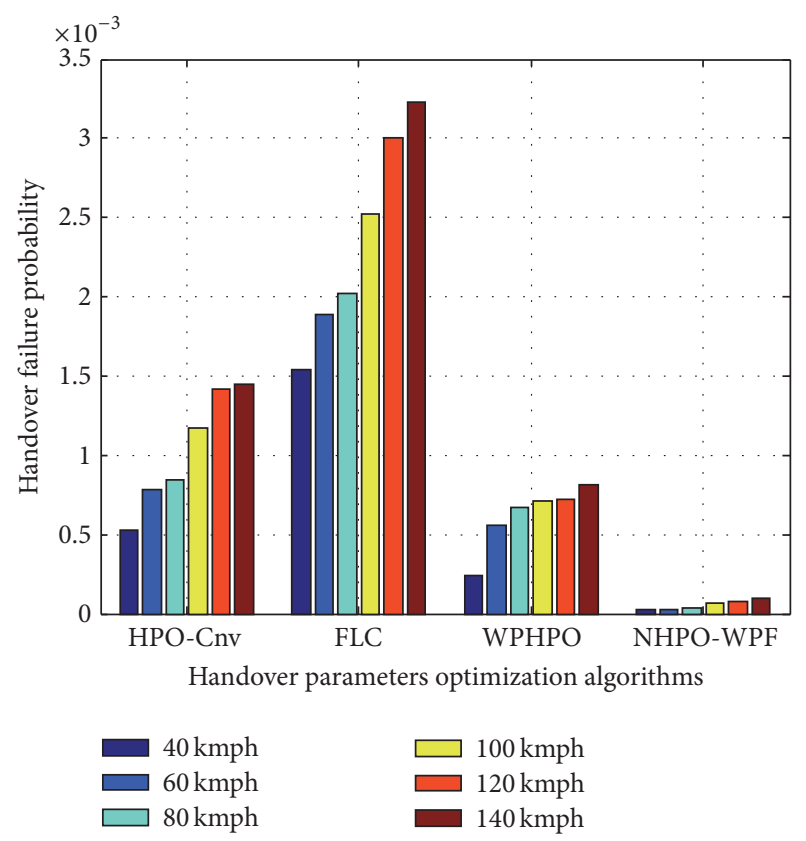

FIGURE 14: Average handover failure probabilities for different mobile speeds based on different mobility handover optimization algorithms.

average reductions of approximately $99.4,99.8$, and $98.6 \%$ compared to the conventional HPO, FLC, and WPHPO algorithms, respectively.

Figure 14 shows the average HFPs from the NHPO-WPF, conventional HPO, FLC, and WPHPO algorithms for the different mobile speeds. The average HFPs were calculated over all the UEs in the system and over all simulation times for each mobile speed scenario independently. The results 
show that the proposed NHPO-WPF algorithm achieves a considerable reduction of HFP compared to the conventional HPO, FLC, and WPHPO algorithms. The average HFPs achieved by NHPO-WPF are approximately 96, 98, and 92\% less than those with the conventional HPO, FLC, and WPHPO algorithms, respectively. An interesting observation is that the results show greater reductions than the reductions of the handover and handover ping-pong probabilities; this is due to the consideration of traffic loads in the optimization process, which leads to estimates of suitable HCPs. This indicates that the resource availability of the target cell is taken into account during the optimization process, which in turn leads to making an accurate handover decision and performing a successful handover as long as the resources are available in the target cell. It will also prevent the serving eNB from making a true handover decision if the target cell does not have sufficient resources; this leads to more successful handovers and decreases the handover failure probability.

The results illustrate that the proposed NHPO-WPF algorithm provides better performance than the conventional HPO, FLC, and WPHPO algorithms. It achieves average reductions of all the handover performance metrics (HOP, HPPP, and HFP) of approximately 96.8, 98.8, and 93.5\% compared to the conventional HPO, FLC, and WPHPO algorithms, respectively. These reductions are mostly due to the effect of the UE's SINR level, velocity, and TL during the estimation of the HCPs; considering these parameters contributed to estimating the appropriate HOM and TTT values, which led to making correct handover decisions. Thus, the proposed NHPO-WPF algorithm based on the CC-CADS scenario achieves significant reductions in HOP, HPPP, and HFP compared to the conventional HPO, FLC, and WPHPO algorithms.

\section{Concluding Remarks}

In this paper, two proposed solutions, known as the CCCADS and the NHPO-WPF algorithm, were introduced and validated. Both solutions enhanced the system performance when they were applied to CA technique in the LTEAdvanced environment. The simulation results showed that CC-CADS provided wider coverage and achieved significant enhancements compared to the standard CADSs, especially at the cell edge. CC-CADS achieved average UE's spectral efficiency improvements of $35 \%, 35 \%$, and $10 \%$ over those of CADS-1, CADS-3, and CADS-3, respectively, and reduced outage probabilities by approximately $49.6 \%, 50 \%$, and $30.6 \%$ compared to CADS-1, CADS-3, and CADS-3, respectively. The proposed NHPO-WPF algorithm provided significant enhancements compared to the conventional HPO algorithm and other algorithms from the literature. The NHPO-WPF algorithm provided average reductions in HOP, HPPP, and HFP of approximately $96.8 \%, 98.8 \%$, and $93.5 \%$ compared to the conventional HPO, FLC, and WPHPO algorithms, respectively. Thus, the two proposed solutions provided better performance than the other considered scenarios and algorithms.

\section{Nomenclature}

List of Terminologies Used in the Paper

\begin{tabular}{|c|c|}
\hline 3GPP: & Third-Generation Partnership Project \\
\hline AMC: & Adaptive Modulation and Coding \\
\hline AWF: & $\begin{array}{l}\text { Automatic proposed weight estimator } \\
\text { function }\end{array}$ \\
\hline CA: & Carrier aggregation \\
\hline CADSs: & $\begin{array}{l}\text { Carrier Aggregation Deployment } \\
\text { Scenarios }\end{array}$ \\
\hline CC-CADS: & $\begin{array}{l}\text { Coordinated Contiguous-Carrier } \\
\text { Aggregation Deployment Scenarios }\end{array}$ \\
\hline CCs: & Component carriers \\
\hline CDF: & Cumulative Distribution Function \\
\hline CDR: & Call Drop Rate \\
\hline CP: & Cyclic Prefix \\
\hline CR: & Coding Rate \\
\hline DCP: & Drop Call Probability \\
\hline DL: & Downlink \\
\hline eNB: & Evolved Node B \\
\hline FLC: & Fuzzy Logic Controller \\
\hline FRF: & Frequency Reuse Factor \\
\hline HCPs: & Handover Control Parameters \\
\hline HFP: & Handover failure probability \\
\hline HOM: & Handover margin \\
\hline HOR: & Handover Ratio \\
\hline HPI: & Handover Performance Indicator \\
\hline HPO: & Handover Parameters Optimization \\
\hline HPPP: & Handover ping-pong probability \\
\hline LTE-Advanced: & Long Term Evolution Advanced \\
\hline MR: & Measurement Reports \\
\hline MS: & Modulation schemes \\
\hline NAS: & Non-Access Stratum \\
\hline NHPO-WPF: & $\begin{array}{l}\text { Novel Handover Parameters Optimization } \\
\text { algorithm that is based on the Weight } \\
\text { Performance Function }\end{array}$ \\
\hline OFDMA: & $\begin{array}{l}\text { Orthogonal Frequency-Division Multiple } \\
\text { Access }\end{array}$ \\
\hline PRB: & Physical Resource Block \\
\hline RG: & Resource grids \\
\hline RLF: & Radio Link Failure \\
\hline RRC: & Radio Resource Control \\
\hline SINR: & Signal-to-Interference Noise Ratio \\
\hline SO: & Self-Optimization \\
\hline TTI: & Transmission Time Interval \\
\hline TTT: & Time-To-Trigger \\
\hline UE: & User Equipment \\
\hline WPF: & Weight Performance Function \\
\hline WPHPO: & $\begin{array}{l}\text { Weighted Performance based on } \\
\text { Handover Parameter Optimizatio }\end{array}$ \\
\hline
\end{tabular}

List of Notations Used in the Paper

$N_{\mathrm{RB}}^{\mathrm{DL}}$ : Total number of DL PRBs over one resource grid

$\overline{P_{\mathrm{HO}}}$ : Average number of handovers per UE

$A_{\mathrm{HPPP}}$ : Average handover ping-pong probability per UE 
$B_{\mathrm{RB}}$ : $\quad$ PRB's bandwidth

$B_{\mathrm{bit}}^{\mathrm{RB}}$ : $\quad$ Total number of bits over each PRB

$B_{\text {bit }}^{\text {sc }}: \quad$ Total number of bits over each subcarrier

$B_{\mathrm{bit}}^{\mathrm{UE}}$ : Total number of useful bits transmitted to UE

$G_{\mathrm{TX}_{m}}: \quad$ Transmitter antenna gain over $\mathrm{CC}_{m}[\mathrm{~dB}]$

$G_{\mathrm{RX}}: \quad$ Receiver antenna gain $[\mathrm{dB}]$

$I_{m, k}: \quad$ Total interferences received signals power on subcarrier $k$ over $\mathrm{CC}_{m}$ from all neighboring eNBs

$L_{S}: \quad$ The occupant serving traffic load

$L_{T}: \quad$ The occupant target traffic load

$L_{\text {max }}$ : Maximum load capacity of the system

$M_{\text {Avg }}$ : Average handover margin level

$M_{\max }$ : Maximum handover margin

$M_{\text {min }}$ : Minimum handover margin

$N_{\mathrm{CC}}^{\mathrm{UE}}$ : Total number of CCs paired to one UE

$N_{\mathrm{FHP}}^{\text {Totl }}$ : Total number of handover failure ratios

$N_{\mathrm{HPP}}^{\text {sys }}$ : Total number of handover ping-pongs overall the system

$N_{\text {No-HPP }}^{\text {sys }}$ Total number of non-ping-pongs

$N_{\mathrm{RB}}^{\text {Total DL}}$ : Total number of available DL PRBs over the entire system bandwidth

$N_{\mathrm{RB}}^{\mathrm{UE}}$ : Total number of PRBs paired to one UE

$N_{\mathrm{RHP}}^{\text {sys }}$ : Total number of requested handovers

$N_{\mathrm{RS}}^{\mathrm{RB}}$ : $\quad$ Total number of resource elements that are configured as reference symbols

$N_{\text {SHP }}^{\text {sys }}$ :

$N_{\text {UEs }}^{\text {sys }}$ :

$N_{\mathrm{sC}}^{\mathrm{CC}}$ :

$N_{\mathrm{sc}}^{\mathrm{RB}}$ :

$N_{\mathrm{sc}}^{\mathrm{UE}}$ :

Total number of successful handovers

Total number of active UEs in the system

Total number of subcarriers per CC

Total number of DL subcarriers over one PRB

$N_{\text {symb }}^{\mathrm{DL}}$ : Total number of DL symbols over one resource grid

$N_{\text {symb }}^{\text {sc }}$ : Total number of modulation symbols over one subcarrier

$P_{\mathrm{RX}_{(m, k)}}$ : UE's received signal power on subcarrier $k$ over $\mathrm{CC}_{m}[\mathrm{dBm}]$

$P_{\mathrm{TX}_{(m, k)}}$ : Transmitted signal power on subcarrier $k$ over $\mathrm{CC}_{m}[\mathrm{dBm}]$

$P_{\text {int }_{(k, m h)}}$ : Interference received signal power by the UE on subcarrier $k$ over $\mathrm{CC}_{m}$ from the neighboring eNB $h$

$P_{\mathrm{HO}}: \quad$ Handover probability

$P_{\mathrm{HO}}(j):$ Handover probability for $\mathrm{UE}_{j}$

$P_{\text {HPPP }}$ : Handover ping-pong probability

$\mathrm{PL}_{m}$ : $\quad$ Path loss over $\mathrm{CC}_{m}[\mathrm{~dB}]$

$P_{\mathrm{no}_{m, k}}$ : The noise power for the UE on subcarrier $k$ over $\mathrm{CC}_{m}$

$P_{\mathrm{TX}}: \quad$ Total transmission power from the eNB over each CC

$R_{\mathrm{bit}}^{\mathrm{UE}}$ : $\quad$ Total number of bits received at UE within a period of $T$
SINR $_{m, k}: \quad$ SINR at the UE on subcarrier $k$ over $\mathrm{CC}_{m}$

$T_{\text {Handed back }}$ : Time taken for the UE to be handed back to the serving eNB

$T_{\text {Interval }}$ : The time interval between the UE leaving the serving $\mathrm{eNB}$ and being returned to the same eNB

$T_{\text {Leave }}$ : Time taken for the UE to leave the serving eNB-A

$T_{\text {critical }}: \quad$ Critical interval

$T_{j}$ : The data bits received time for the $\mathrm{UE}_{j}$

$T_{\max }: \quad$ Maximum TTT interval

$T_{\min }: \quad$ Minimum TTT interval

$f_{\text {WPF }}(\gamma, L, v)$ : Weight Performance Function

$f_{c}: \quad$ Carrier frequency

$m_{\text {bit }}^{\text {symb }}: \quad$ Total number of bits over one modulation symbol

$P_{\mathrm{TX}_{(m, k)}}: \quad$ Total transmission power over each subcarrier in watt

$v_{\max }: \quad$ Maximum expected UE's velocity

$x_{1}: \quad \gamma$

$x_{2}: \quad L$

$x_{3}: \quad v$

$\beta_{S}: \quad$ Serving signal level

$\beta_{T}: \quad$ Target signal level

$\gamma_{S}$ : $\quad$ SINR over the serving PCC

$\gamma_{T}: \quad$ Target SINR

$\gamma_{\text {Thr }}: \quad$ SINR threshold level

$\gamma_{\max }: \quad$ Maximum SINR

$\eta_{j}: \quad$ Spectral efficiency $\eta_{j}$ for $\mathrm{UE}_{j}$

$\psi_{\mathrm{dB}}$ : Log-normal shadowing in $\mathrm{dB}$

$\omega_{\mathrm{BW}}^{\mathrm{UE}}: \quad \quad \quad \quad \quad \quad$ llocated bandwidth to one UE

$\omega_{L}$ : Weights of traffic load bounded function $f(L)$

$\omega_{\text {sinr }}: \quad$ Weights of SINR bounded function $f(\gamma)$

$\omega_{v}: \quad$ Weights of velocity bounded function $f(v)$

$\omega_{x}$ : Weight of function $f(x)$

$\vartheta_{\mathrm{dB}}: \quad$ Rayleigh fast fading effect in $\mathrm{dB}$

$\Delta T: \quad$ The update interval in TTT

$h$ : Neighboring eNB's number

$j: \quad$ UE's number

$m: \quad$ CC's number

$P_{\text {out }}: \quad$ Outage probability

T310: $\quad$ Maximum interval to perform connection reestablishment procedure

E: $\quad$ Code rate

$F$ : Optimizing parameters factor

$H: \quad$ Total number of neighboring eNBs located in the first tier around the served eNB

L: $\quad$ Traffic load

M: $\quad$ Handover margin level

PL: $\quad$ Path loss

U: $\quad$ Total system component carries

$d: \quad$ Distance

$f\left(x_{i}\right): \quad$ Bounded function, where $x_{i}$ can be $\gamma, L$, or

$f(L): \quad$ Traffic load bounded function

$f(v)$ : $\quad$ Velocity bounded function 
$f(x)$ : Bounded function, which can be $f(\gamma)$, $f(L)$, or $f(v)$

$f(\gamma)$ : SINR bounded function

$k$ : Subcarrier's number

$v: \quad$ UE's velocity

Q: $\quad$ Optimization step level

Z1: The update interval of TTT toward the maximum and minimum TTT interval

Z2: The update interval of TTT toward the maximum TTT interval only

Z3: The update interval of TTT toward the minimum TTT interval only

$\gamma: \quad$ SINR

$\rho: \quad$ Optimization interval

$\tau: \quad$ Time.

\section{Competing Interests}

The authors declare that they have no competing interests.

\section{Acknowledgments}

The authors acknowledge the financial contribution from Grant nos. 01-01-02-SF0789 (MOSTI) and GUP-2012-036 for the publication of this work.

\section{References}

[1] 3GPP, "Carrier aggregation deployment scenarios," Tech. Rep. R2-100531, NTT DOCOMO, Valencia, Spain, 2010.

[2] 3GPP, Simulation assumptions for Mobility performance in Carrier Aggregation, R4-102114, NTT DOCOMO Montreal, 2010, http://www.3gpp.org/.

[3] M. Iwamura, K. Etemad, M.-H. Fong, R. Nory, and R. Love, "Carrier aggregation framework in 3GPP LTE-advanced," IEEE Communications Magazine, vol. 48, no. 8, pp. 60-67, 2010.

[4] I. Shayea, M. Ismail, and R. Nordin, "Capacity evaluation of Carrier Aggregation techniques in LTE-Advanced system," in Proceedings of the International Conference on Computer and Communication Engineering (ICCCE '12), pp. 99-103, IEEE, Kuala Lumpur, Malaysia, July 2012.

[5] 3GPP, Self-configuring and self-optimizing network use cases and solutions, (LTE (Release 10)), TR 36.902 V1.2.0, 2009, http://www.3gpp.org/.

[6] 3GPP, "Feasibility study for Further Advancements for EUTRA (LTE-Advanced(Release 10))," TR 36.912 V10.0.0, 2011, http://www.3gpp.org/.

[7] 3GPP, "Feasibility study for Further Advancements for EUTRA (LTE-Advanced (Release 11))," TR 36.912 V11.0.0, 2012, http://www.3gpp.org/.

[8] 3GPP, "Technical Specification Group Services and System Aspects; Telecommunication Management; Self-Organizing Networks (SON) Policy Network Resource Model (NRM) Integration Reference Point (IRP); Requirements (Release 11)," TS 32.521 V11.1.0, 2014, http://www.3gpp.org/.

[9] 3GPP, “Technical Specification Group Services and System Aspects; Telecommunication Management; Self-Organizing Networks (SON) Policy Network Resource Model (NRM) Integration Reference Point (IRP); Requirements (Release 12)," TS 28.627 V12.0.0, 2014, http://www.3gpp.org/.
[10] 3GPP, “Technical Specification Group Services and System Aspects; Telecommunication management; Self-Organizing Networks (SON) Policy, Network Resource Model (NRM), Integration Reference Point (IRP); Information Service (IS) (Release 12)," TS 28.628 V12.1.0, 2014, http://www.3gpp.org/.

[11] 3GPP, “Technical Specification Group Radio Access Network; Evolved Universal Terrestrial Radio Access (E-UTRA); UE Equipment (UE) procedures in idle mode (Release 12)," TS 36.304 V12.3.0., 2014, http://www.3gpp.org/.

[12] I. M. Bălan, B. Sas, T. Jansen, I. Moerman, K. Spaey, and P. Demeester, "An enhanced weighted performance-based handover parameter optimization algorithm for LTE networks," EURASIP Journal on Wireless Communications and Networking, vol. 2011, no. 1, article 98, pp. 1-11, 2011.

[13] T. Jansen, I. Balan, J. Turk, I. Moerman, and T. Kürner, "Handover parameter optimization in LTE self-organizing networks," in Proceedings of the IEEE 72nd Vehicular Technology Conference Fall (VTC '10), pp. 6-9, September 2010.

[14] P. Munoz, R. Barco, and I. de la Bandera, "On the potential of handover parameter optimization for self-organizing networks," IEEE Transactions on Vehicular Technology, vol. 62, no. 5, pp. 1895-1905, 2013.

[15] H. Zhu and K.-S. Kwak, "Performance analysis of an adaptive handoff algorithm based on distance information," Computer Communications, vol. 30, no. 6, pp. 1278-1288, 2007.

[16] 3GPP, “Technical Specification Group Radio Access Network; E-UTRA and E-UTRAN; Over all description; Stage 2 (Release 12)," TS 36.300 V12.4.0., 2014, http://www.3gpp.org/.

[17] 3GPP, "E-UTRA; Radio Frequency (RF) system scenarios (Release 12)," 3GPP TR 36.942 V12.0.0, 2014, http://www.3gpp .org/.

[18] 3GPP, “(E-UTRA); Physical channels and modulation (Release 12),” TS 36.211 V12.4.0., 2014, http://www.3gpp.org/.

[19] 3GPP, “(E-UTRA); LTE physical layer; General description (Release12)," TS 36.201 V12.1.00, 2012, http://www.3gpp.org/.

[20] 3GPP, “(E-UTRA); physical layer procedures (release 12)," Tech. Rep. TS 36.213 V12.4.0, 2014, http://www.3gpp.org/.

[21] 3GPP, "Evolved Universal Terrestrial Radio Access (E-UTRA); Radio Resource Control (RRC); Protocol specification (Release 11)," Valbonne-FRANCE, TS 36.331 V11.10.0., 2014, http://www.3gpp.org/.

[22] 3GPP, "Evolved Universal Terrestrial Radio Access (E-UTRA); Requirements for support of radio resource management (Release 11)," Valbonne-France, TS 36.133 V11.11.0, 2014, http://www.3gpp.org/.

[23] P. J. Song and J. Shin, "Method for Handover in Multi-Carrier System," Google Patents, 2011.

[24] L. Zhang, K. Zheng, W. Wang, and L. Huang, "Performance analysis on carrier scheduling schemes in the long-term evolution-advanced system with carrier aggregation," IET Communications, vol. 5, no. 5, pp. 612-619, 2011.

[25] E. Dahlman, P. Stefan, and S. Johan, 4G: LTE/LTE-Advanced for Mobile Broadband, Academic Press, Cambridge, Mass, USA, 2nd edition, 2014.

[26] I. Shayea, M. Ismail, and R. Nordin, "Downlink spectral efficiency evaluation with carrier aggregation in LTE-Advanced system employing Adaptive Modulation and Coding schemes," in Proceedings of the IEEE 11th Malaysia International Conference on Communications (MICC '13), pp. 98-103, IEEE, Kuala Lumpur, Malaysia, November 2013. 
[27] G. P. Pollini, “Trends in handover design,” IEEE Communications Magazine, vol. 34, no. 3, pp. 82-90, 1996.

[28] V. Garcia, N. Lebedev, and J.-M. Gorce, "Capacity outage probability for multi-cell processing under Rayleigh fading," IEEE Communications Letters, vol. 15, no. 8, pp. 801-803, 2011.

[29] J. F. Paris and D. Morales-Jiménez, "Outage probability analysis for Nakagami-q (Hoyt) fading channels under Rayleigh interference," IEEE Transactions on Wireless Communications, vol. 9, no. 4, pp. 1272-1276, 2010. 

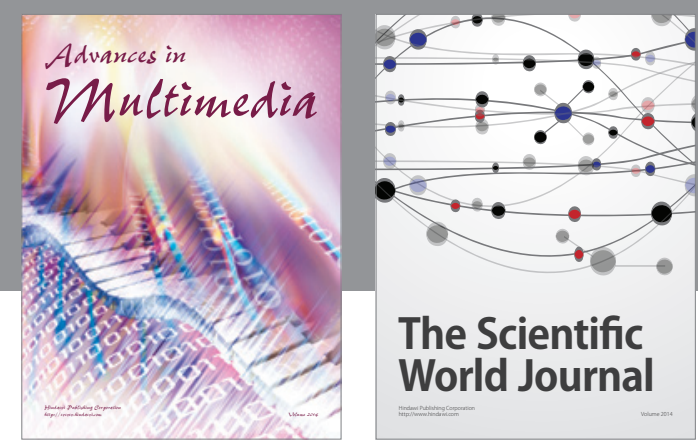

The Scientific World Journal
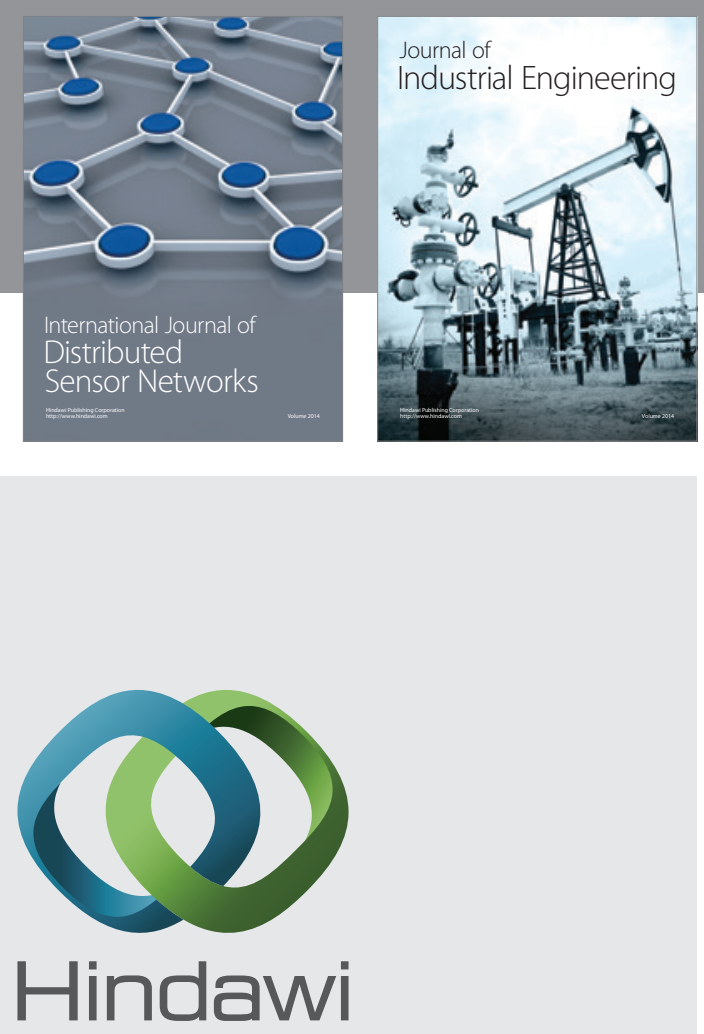

Submit your manuscripts at

http://www.hindawi.com

\section{Computer Networks} and Communications
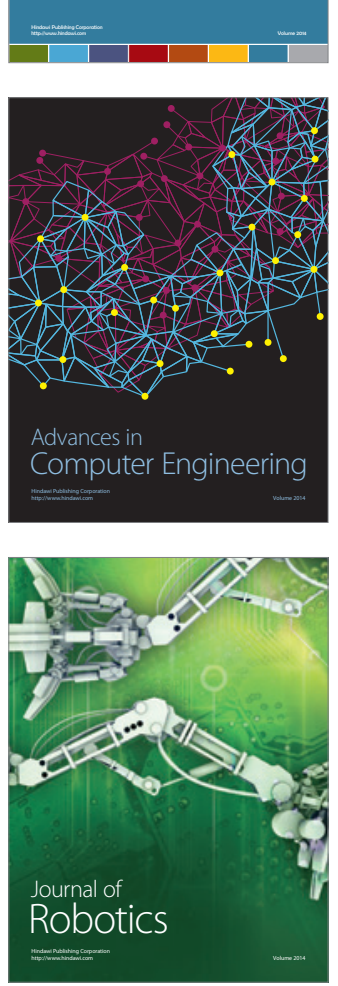
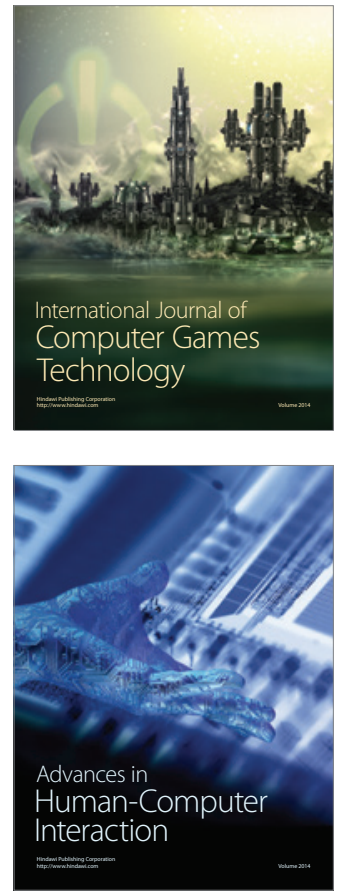
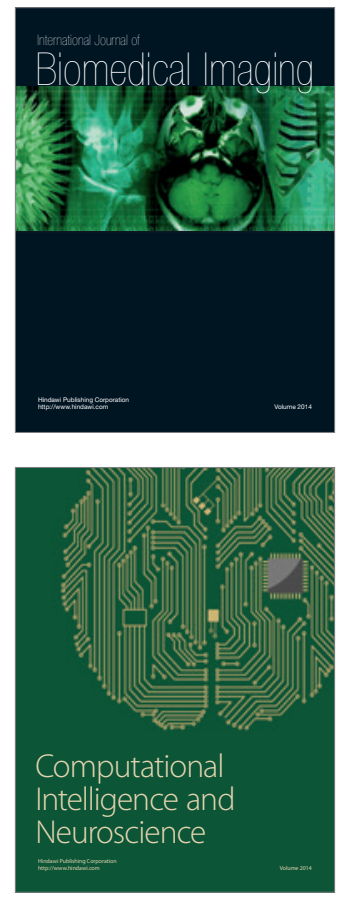
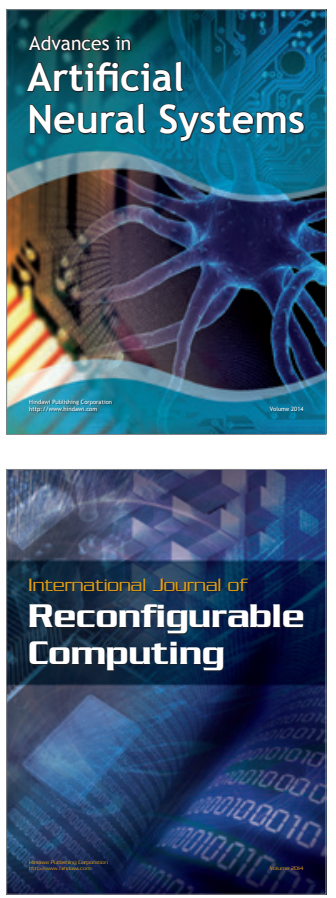
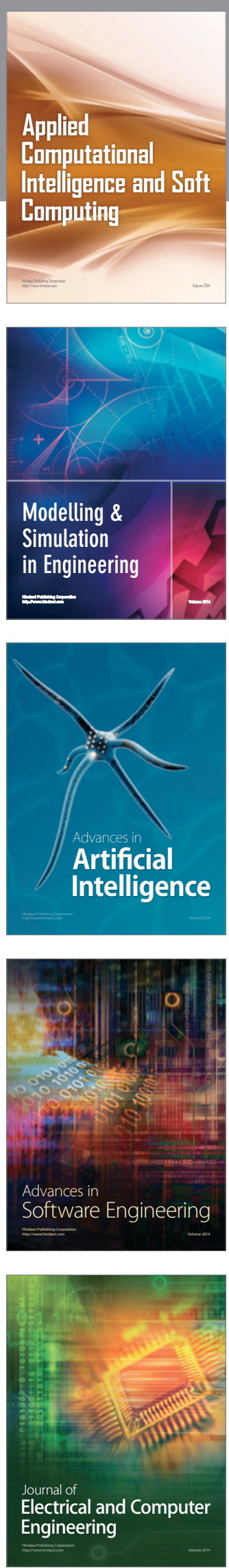